\title{
Precision spectroscopy and higher spin symmetry in the ABJM model
}

\author{
Massimo Bianchi, ${ }^{a, b}$ Rubik Poghossian ${ }^{a, c}$ and Marine Samsonyan ${ }^{a}$ \\ a Dipartimento di Fisica, Università di Roma "Tor Vergata", \\ I.N.F.N. Sezione di Roma "Tor Vergata", \\ Via della Ricerca Scientifica, 00133 Roma, Italy \\ ${ }^{b}$ Physics Department, Theory Unit, CERN, \\ CH 1211, Geneva 23, Switzerland \\ ${ }^{c}$ Yerevan Physics Institute, \\ Alikhanian Br. 2, 0036 Yerevan, Armenia \\ E-mail: massimo.bianchi@roma2.infn.it, \\ Marine.Samsonyan@roma2.infn. it, Rubik.Poghosyan@roma2.infn.it
}

ABSTRACT: We revisit Kaluza-Klein compactification of 11-d supergravity on $S^{7} / \mathbb{Z}_{k}$ using group theory techniques that may find application in other flux vacua with internal coset spaces. Among the $\mathrm{SO}(2)$ neutral states, we identify marginal deformations and fields that couple to the recently discussed world-sheet instanton of Type IIA on $\mathbb{C P}^{3}$. We also discuss charged states, dual to monopole operators, and the $\mathbb{Z}_{k}$ projection of the $O s p(8 \mid 4)$ singleton and its tensor products. In particular, we show that the doubleton spectrum may account for $\mathcal{N}=6$ higher spin symmetry enhancement in the limit of vanishing 't Hooft coupling in the boundary Chern-Simons theory.

KEYwORDS: AdS-CFT Correspondence, M-Theory

ARXIV EPRINT: 1005.5307 


\section{Contents}

1 Introduction $\quad 1$

2 The ABJM model 2

2.1 Supergravity description 2

2.2 Boundary CFT description 3

2.3 A quick look at the spectrum 4

3 Compactification on $S^{7}$ revisited $\quad 5$

4 Polynomial representations for $\mathrm{SO}(8)$ and $\mathrm{U}(4) \quad 9$

5 From $S^{\mathbf{7}}$ to $\mathbb{C P}^{\mathbf{3}} \ltimes S^{\mathbf{1}} \quad \mathbf{1 3}$

$\begin{array}{lll}5.1 \text { A closer look at the KK spectrum } & 15\end{array}$

6 Singleton, partition functions and higher spins $\quad 17$

$\begin{array}{ll}\text { 6.1 Doubleton and higher spin gauge fields } & 18\end{array}$

6.2 Tripletons and higher $n$-pletons 22

$\begin{array}{lll}6.3 & \text { KK excitations } & 26\end{array}$

$\begin{array}{llr}7 & \text { Conclusions } & 28\end{array}$

$\begin{array}{ll}\text { A Dimension formulae for } \mathrm{SO}(8) & 28\end{array}$

$\begin{array}{ll}\text { B Zero charge states } & 29\end{array}$

C Generating functions for $\mathrm{SO}(8)$ representations 30

D Generating functions for $\mathrm{SO}(6)$ representations 31

\section{Introduction}

The spectrum of Kaluza-Klein (KK) excitations in flux vacua plays an important role both in attempts to embed the Standard Model in String Theory and in the holographic correspondence. In the spirit of holography, the seminal observation of Schwarz's [1] and the subsequent work of Bagger and Lambert [2-5] and, independently, of Gustavsson [6], motivated Aharony, Bergman, Jafferis and Maldacena (ABJM) $[7,8]$ to propose a duality between superconformal Chern-Simons (C-S) theories in $d=3$ dimensions and String / Mtheory on $A d S_{4}$. 
The duality has been thoroughly tested and extended to cases with lower supersymmetry [9-14]. In particular the superconformal index has been matched both in the regime $k \gg 1$ (SO(2) singlets) $[15,16]$ and at finite $k[17,18]$. A detailed analysis of the (BPS) spectrum and the supermultiplet structure is however still incomplete. Aim of this note is to fill in this gap and perform precision spectroscopy of 11-d supergravity on $A d S_{4} \times S^{7} / \mathbb{Z}_{k}$ or, equivalently, Type IIA on $A d S_{4} \times \mathbb{C P}^{3}$. We will also discuss higher spin symmetry enhancement in the limit of vanishing 't Hooft coupling in the boundary $\mathcal{N}=6$ ChernSimons theory.

The plan of the paper is as follows. After reviewing the ABJM model, presenting both bulk and boundary vantage points, we will revisit KK reduction of 11-d supergravity on $S^{7}[19]$ and then perform the decomposition of $\mathrm{SO}(8)$ into $\mathrm{SO}(6) \times \mathrm{SO}(2)$ so as to derive the KK excitations of $\mathcal{N}=6$ gauged supergravity [20], including states charged under $\mathrm{SO}(2)$ that are expected to be dual to 'monopole' operators on the boundary $[7,8]$. Since we rely on group theory techniques which are not easily found in the available literature, we try to make this part of the presentation as pedagogical as possible, also in view of applications to other flux vacua with internal coset manifolds $G / H$. We then compare the resulting bulk spectrum with the spectrum of gauge-invariant operators on the boundary. Finally we compute the partition function of the boundary theory performing an orbifold projection on the parent theory ( $k=1,2$ cases) and examine the higher spin content of the theory. Various appendices summarize useful $\mathrm{SO}(8)$ and $\mathrm{SO}(6)$ group theory formulae.

\section{The ABJM model}

The near-horizon geometry of a stack of $N$ M2-branes is $A d S_{4} \times S^{7}$ with $N$ units of $F_{4}$ flux along $A d S_{4}$ and as many units of its dual $F_{7}$ along $S^{7}$ [21]. The metric reads

$$
d s_{11}^{2}=\frac{1}{4} L^{2} d s_{\text {AdS }}^{2}+L^{2} d s_{S^{7}}^{2}
$$

for later use, note that $L_{\mathrm{AdS}}=L / 2$ with $L$ the radius of $S^{7}$ and henceforth the metrics of the subspaces are for unit curvature radii.

ABJM have conjectured that 11-d supergravity on $A d S_{4} \times S^{7} / \mathbb{Z}_{k}$, corresponding to the near horizon geometry of $N$ M2-branes at a $\mathbb{C}^{4} / \mathbb{Z}_{k}$ singularity, be dual to $\mathcal{N}=6$ C-S theory in $d=3$ with gauge group $\mathrm{U}(N)_{k} \times \mathrm{U}(N)_{-k}$ and opposite CS couplings $k_{1}=k=-k_{2}[7,8]$.

\subsection{Supergravity description}

The Type IIA solution corresponding to the ABJM model reads

$$
d s_{I I A}^{2}=4 \frac{\rho^{2}}{L^{2}} d x \cdot d x+4 \frac{L^{2}}{4 \rho^{2}} d \rho^{2}+L^{2} d s_{\mathbb{C P}^{3}}^{2}=\frac{1}{4} L^{2} d s_{\mathrm{AdS}}^{2}+L^{2} d s_{\mathbb{C P}^{3}}^{2}
$$

where

$$
L=\left(\frac{32 \pi^{2} N}{k}\right)^{1 / 4}
$$


is the curvature radius in string units. The string coupling, related to the VEV of the dilaton, is given by

$$
g_{s}=L / k=\left(\frac{32 \pi^{2} N}{k^{5}}\right)^{1 / 4}
$$

Thus the perturbative Type IIA description should be valid for $L \gg 1$ and $g_{s} \ll 1$ i.e. for $N^{1 / 5} \ll k \ll N$ while $\lambda=N / k$ is the 't Hooft coupling of the boundary CS theory.

In the 11-d uplift, $\mathbb{C P}^{3}$ becomes the base of a Hopf fibration $S^{7}=\mathbb{C P}^{3} \ltimes S^{1}$ whose metric reads

$$
d s_{S^{7}}^{2}=d s_{\mathbb{C P}^{3}}^{2}+(d \tau+\mathcal{A})^{2}
$$

with $d \mathcal{A}=2 \mathcal{J}_{\mathbb{C P}^{3}}$, the Kähler form on $\mathbb{C P}^{3}$ normalized so that $d V\left(\mathbb{C P}^{3}\right)=\mathcal{J} \wedge \mathcal{J} \wedge \mathcal{J} / 6$ and $V\left(\mathbb{C P}^{3}\right)=\pi^{3} / 6$. The solution is supported by R-R fluxes

$$
g_{s} F_{2}=2 L \mathcal{J} \quad, \quad g_{s} F_{4}=6 L^{3} d V\left(A d S_{4}\right) \quad, \quad g_{s} F_{6}=6 L^{5} d V\left(\mathbb{C P}^{3}\right)
$$

In the ABJM model, corresponding to $\mathcal{N}=6$ C-S theory $\mathrm{U}(N)_{k} \times \mathrm{U}(N)_{-k}$ on the boundary, $B_{2}=0$. For fractional M2-branes, one has the ABJ model corresponding to $\mathcal{N}=6$ C-S theory $\mathrm{U}(N)_{k} \times \mathrm{U}(N+k-l)_{-k}[22]$ on the boundary, $B_{2}=\mathcal{J} l / k$, with $l=1, \ldots, k-1$. Boundary C-S theories with $\sum_{i} k_{i} \neq 0$ and lower susy should be dual to turning on a non-zero Romans mass $\left(F_{0} \neq 0\right)$ in the bulk Type IIA description [23-25].

The 11-d supergravity approximation should be valid in the double-scaling limit $k \rightarrow$ $\infty, N \rightarrow \infty$ with $\lambda=N / k$ fixed and large. The CFT description, to which we momentarily turn our attention, should instead be valid when $\lambda \ll 1$, i.e. $k \gg N$. As $\lambda \rightarrow 0$ higher spin symmetry enhancement takes place as we will eventually see.

\subsection{Boundary CFT description}

$\mathcal{N}=6$ CS theories are conveniently constructed from $\mathcal{N}=3$ CS theories. The case $\mathcal{N}=3$ arises in turn from the $\mathcal{N}=4$ case obtained after dimensional reduction of $\mathcal{N}^{\prime}=2$ in $d=4$. In this way, each vector multiplet includes an $\mathcal{N}=2$ (i.e. $\mathcal{N}=1^{\prime}$ in $d=4$ ) chiral multiplet in the adjoint $\Phi=\Phi_{a} t^{a}$ and couples to various hypers $Q$ and $\tilde{Q}$ in real (reducible) representations. Adding to the 'standard' $\mathcal{N}=4$ superpotential

$$
W=\tilde{Q} \Phi Q
$$

the CS term, giving a mass $m=g_{Y M}^{2} \frac{k}{4 \pi}$ to the vectors, and a CS superpotential

$$
W=\frac{k}{8 \pi} \operatorname{Tr} \Phi^{2}
$$

breaks $\mathcal{N}=4$ to $\mathcal{N}=3$. Integrating out $\Phi$ yields

$$
W=\frac{4 \pi}{k}\left(\tilde{Q} t^{a} Q\right)\left(\tilde{Q} t^{a} Q\right)
$$


The resulting $\mathcal{N}=3$ theory has no marginal susy preserving deformations [23-25]. In the process R-symmetry is reduced to $\mathrm{SO}(3) \approx \mathrm{SU}(2)$ for $\mathcal{N}=3$ from the original $\mathrm{SO}(4)$ of $\mathcal{N}=4$.

The case $\mathcal{N}=6$ is special. Starting with the $\mathcal{N}=3$ theory with $G=\mathrm{U}(N)_{k} \times \mathrm{U}(N)_{-k}$ and two pairs of hypers, $A_{r} \in\left(\mathbf{N}, \mathbf{N}^{*}\right)$ and $B_{\dot{m}} \in\left(\mathbf{N}^{*}, \mathbf{N}\right)$ and integrating out $\Phi_{1}$ and $\Phi_{2}$ one gets

$$
W=\frac{2 \pi}{k} \epsilon^{r s} \epsilon^{\dot{m} \dot{n}} \operatorname{Tr}\left(A_{r} B_{\dot{m}} A_{s} B_{\dot{n}}\right)
$$

Since the manifest 'flavour' symmetry of $W$ under $\mathrm{SU}(2) \times \mathrm{SU}(2) \times \mathrm{U}(1)_{B}$ does not commute with R-symmetry $\mathrm{SO}(3) \approx \mathrm{SU}(2)$ under which $A$ and $B$ form doublets, the full theory has a larger $\mathrm{SU}(4) \approx \mathrm{SO}(6)$ symmetry which is the R-symmetry of $\mathcal{N}=6$. To expose the symmetry it is convenient to define $X^{i}=\left(A_{1}, A_{2}, B_{\dot{1}}^{*}, B_{\dot{2}}^{*}\right)$ and their conjugate $X_{i}^{*}$ that together transform as $\mathbf{4}_{+1}+\mathbf{4}_{-1}^{*}$ of $\mathrm{SO}(6) \times \mathrm{SO}(2)$. As we will momentarily see, $\mathrm{SO}(2) \sim \mathrm{U}(1)$ acts as a baryonic symmetry. Further (super)symmetry enhancement to $\mathcal{N}=8$ with $\mathrm{SO}(8) \mathrm{R}$-symmetry takes place for $k=1$ and $k=2$. The former corresponds to compactification on $S^{7}$ the latter to $S^{7} / Z_{2}$ (only 'even' spherical harmonics).

\subsection{A quick look at the spectrum}

The (ungauged) $\mathcal{N}=6$ supergravity multiplet consists of the graviton $g_{\mu \nu}, 6$ gravitini $\psi_{\mu}^{i}$, 16 graviphotons $A_{\mu}^{[i j]}$ and $A_{\mu}^{0}, 26$ dilatini $\lambda^{[i j k]}$ and $\lambda_{i}$, and 30 scalars $\phi^{[i j k l]}$ and $\phi_{[i j]}$. The latter parameterize the moduli space $\mathcal{M}=S O^{*}(12) / \mathrm{U}(6)$. After 'gauging' $\mathrm{SO}(6) \times \mathrm{SO}(2)$ a scalar potential is generated and the two sets of $\mathbf{1 5} \mathbf{5}_{0}$ scalars become 'massive' or rather 'tachyonic' i.e. $\left(M L_{\mathrm{AdS}}\right)^{2}=-2$, safely above the B-F bound $\left(M L_{\mathrm{AdS}}\right)^{2}=-9 / 4$.

Compactification of Type IIA supergravity on $\mathbb{C P}^{3}$ was studied in [26]. KK excitations with $Q=0$, i.e. neutral wrt $\mathrm{SO}(2)$, were identified there. The non-perturbative spectrum, contains various wrapped branes, including D0-branes that are charged wrt $\mathrm{SO}(2)$. The latter correspond to 11-d KK modes along the compact circle that can be obtained by a $\mathbb{Z}_{k}$ projection of the M-theory compactification on $S^{7}$. The dual to $\mathrm{SO}(2)$ charged states are monopole operators on the boundary [8, 27, 28]. Although the fundamental fields $\left(A_{r}, B_{\dot{s}}\right)$ are neutral wrt the diagonal $\mathrm{U}(1)$ that couples to $A_{\mu}^{+}=A_{\mu}^{1}+A_{\mu}^{2}$, the orthogonal combination $A_{\mu}^{-}=A_{\mu}^{1}-A_{\mu}^{2}$ acts as a baryonic symmetry. The corresponding current, $J_{B}=* F^{+}$, is conserved thanks to Bianchi identities. Due to the CS coupling $k \int A^{-} \wedge F^{+}$, configurations with $A^{+}$magnetic charge are electrically charged wrt $A^{-}$. Alternatively one can introduce a Lagrange multiplier $\tau$ for $d F^{+}=0$ (on-shell $k A^{-}=d \tau$ ) and form combinations $e^{i n \tau}$ that can screen the baryonic charge of matter field composites. In general one can consider magnetic monopoles charged under $\mathrm{U}(1)^{N} \subset \mathrm{U}(N)$ with $H=$ $\left(Q_{1}, \ldots, Q_{N}\right)$. Without loss of generality one can take $Q_{1} \geq Q_{2} \geq \ldots \geq Q_{N}$. Since elementary fields have unit charges and transform in the fundamental of $\mathrm{SU}(N)$, these monopole operators correspond to Young tableaux with $k Q_{i}$ boxes in the $i^{\text {th }}$ row. For $k=1,2$ dressing composite vector currents in the $\mathbf{6}_{ \pm 2}$ and scalar operators in the $\mathbf{1 0}_{ \pm 2}$ 
and $10_{\mp 2}^{*}$ (with $\Delta_{ \pm}=1,2$ ) with charge 2 monopole operators is crucial to the enhancement of supersymmetry to $\mathcal{N}=8$ with full $\mathrm{SO}(8)$ R-symmetry [8]. Monopole and anti-monopole operators however appear in the spectrum even when $k \geq 3$ and no (super)symmetry enhancement takes place $[27,28]$.

Before concluding this preliminary look, let us note that out of the two $\mathrm{U}(1)$ in the boundary CS theory only the Baryonic $\mathrm{U}(1)_{B}=\mathrm{U}(1)_{-}$is visible as a global symmetry, whose $\mathbb{Z}_{k}$ subgroup is gauged, in the bulk description. The fate of the other $\mathrm{U}(1)$ is a sort of Higgs mechanism, under which $A_{M} \rightarrow A_{\mu}$ and $C_{M N P} \rightarrow C_{\mu} \mathcal{J}_{a b}$ mix. Only the combination $k A_{\mu}+N C_{\mu}$ remains massless and couples to $\mathrm{U}(1)_{B}$ while the orthogonal combination $N A_{\mu}-k C_{\mu}$ becomes massive by 'eating' the (pseudo)scalar $\beta$ from $B_{2}=\beta \mathcal{J}$. A 5 -brane instanton is thus expected to mediate processes in which $k$ D0-branes transform into $N$ D4-branes wrapped around $C P^{2} \subset \mathbb{C P}^{3}[22]$.

\section{Compactification on $S^{7}$ revisited}

For the later use let us briefly review the mass spectrum of the Freund-Rubin solution of $d=11$ supergravity on $S^{7}[19,29,30]$. The gravitino field as well as all the fermions are set to zero, the $A d S_{4}$ Riemann tensor and the three-form field strength are given by:

$$
\begin{aligned}
& R_{\mu \nu \rho \sigma}=-4\left(g_{\mu \rho}(x) g_{\nu \sigma}(x)-g_{\mu \sigma}(x) g_{\nu \rho}(x)\right) \\
& F_{\mu \nu \rho \sigma}=3 \sqrt{2} \sqrt{-\operatorname{det} g_{\mu \nu}(x)} \varepsilon_{\mu \nu \rho \sigma}
\end{aligned}
$$

where $\varepsilon_{0123}=-1$. The metric and the three form field with mixed indices vanish:

$$
g_{\mu \alpha}=F_{\mu \nu \rho \alpha}=F_{\mu \nu \alpha \beta}=F_{\mu \alpha \beta \gamma}=0
$$

and also

$$
\begin{aligned}
F_{\alpha \beta \gamma \delta}(y) & =0 \\
R_{\alpha \beta} & =-6 g_{\alpha \beta}(y)
\end{aligned}
$$

$\mu, \nu, \rho=0, \ldots, 3$ are $d=4$ indices, $\alpha, \beta, \gamma=1, \ldots, 7$ are internal indices.

Let us then consider fluctuations around the Freund-Rubin solution. The linearized field equations are obtained by replacing the background fields in the $d=11$ field equations by background fields plus arbitrary fluctuations. An elegant and quite general method to determine the complete mass spectrum on any coset manifold relies on generalized harmonic expansion. In our case, one expands the fluctuations in a complete set of spherical harmonics of $S^{7}=\mathrm{SO}(8) / \mathrm{SO}(7)$. The coefficient functions of the spherical harmonics correspond to the physical fields in $d=4$. In order to diagonalize the linearized equations 
it turns out to be convenient to parameterize the fluctuations as follows:

$$
\begin{aligned}
g_{\mu \nu}(x, y) & =g_{\mu \nu}(x)+h_{\mu \nu}(x, y) \\
h_{\mu \nu}(x, y) & =h_{\mu \nu}^{\prime}(x, y)-\frac{1}{2} g_{\mu \nu}(x) h_{\alpha}^{\alpha}(x, y) \\
g_{\alpha \beta}(x, y) & =g_{\alpha \beta}(x)+h_{\alpha \beta}(x, y) \\
g_{\mu \alpha}(x, y) & =h_{\mu \alpha}(x, y) \\
A_{\mu \nu \rho}(x, y) & =A_{\mu \nu \rho}(x)+a_{\mu \nu \rho}(x, y)
\end{aligned}
$$

In particular the Weyl rescaled spacetime metric appears in (3.7) so as to put the $d=4$ Einstein action in canonical form. The spherical harmonic expansions of the fluctuations of the metric and of the antisymmetric tensor fields are given by:

$$
\begin{aligned}
h_{(\mu \nu)}^{\prime}(x, y) & =\sum H_{\mu \nu}^{N_{1}}(x) Y^{N_{1}}(y) \\
h_{\mu \alpha}(x, y) & =\sum B_{\mu}^{N_{7}}(x) Y_{\alpha}^{N_{7}}(y)+B_{\mu}^{N_{1}}(x) D_{\alpha} Y^{N_{1}}(y) \\
h_{(\alpha \beta)}(x, y) & =\sum \phi^{N_{27}}(x) Y_{(\alpha \beta)}^{N_{27}}(y)+\phi^{N_{7}}(x) D_{(\alpha} Y_{\beta)}^{N_{7}}(y)+\phi^{N_{1}}(x) D_{(\alpha} D_{\beta} Y^{N_{1}}(y) \\
h_{\alpha}^{\alpha}(x, y) & =\sum \pi^{N_{1}}(x) Y^{N_{1}}(y) \\
A_{\mu \nu \rho}(x, y) & =\sum a_{\mu \nu \rho}^{N_{1}}(x) Y^{N_{1}}(y) \\
A_{\mu \nu \alpha}(x, y) & =\sum a_{\mu \nu}^{N_{7}}(x) Y_{\alpha}^{N_{7}}(y)+a_{\mu \nu}^{N_{1}}(x) D_{\alpha} Y^{N_{1}}(y) \\
A_{\mu \alpha \beta}(x, y) & =\sum a_{\mu}^{N_{21}}(x) Y_{\alpha \beta}^{N_{21}}(y)+a_{\mu}^{N_{7}}(x) D_{[\alpha} Y_{\beta]}^{N_{7}} \\
A_{\alpha \beta \gamma}(x, y) & =\sum a^{N_{35}}(x) Y_{\alpha \beta \gamma}^{N_{35}}(y)+a^{N_{21}}(x) D_{[\alpha} Y_{\beta \gamma]}^{N_{21}}(y)
\end{aligned}
$$

All superscripts $N_{\mathbf{r}}(\mathbf{r}=1,7,21,27,35)$ have infinite range, since they should provide a basis for arbitrary fields on the 7 -sphere. The index $\mathbf{r}$ specifies the $\mathrm{SO}(7)$ representation of the corresponding spherical harmonic. For example, $Y_{\alpha \beta \gamma}^{N_{35}}$ is in the third rank totally antisymmetric representation of $\mathrm{SO}(7)$ with dimension 35 , while $Y_{(\alpha \beta)}^{N_{27}}$ is in the symmetric traceless 27-dimensional representation. Derivatives of $Y$ 's appear in the expansions since any tensor can be decomposed into its transverse and longitudinal parts. After fixing all local symmetries which do not correspond to gauge invariances of the final $d=4$ theory and by choosing de Donder type, $D^{\alpha} h_{(\alpha \beta)}(x, y)=0$, and Lorentz type, $D^{\alpha} h_{\alpha \mu}(x, y)=0$, conditions the last term in $h_{\mu \alpha}$ and the last two terms in $h_{(\alpha \beta)}$ drop out. To fix the local symmetries of the antisymmetric tensor fields we choose the Lorentz conditions $D^{\alpha} A_{\alpha \beta \gamma}(x, y)=D^{\alpha} A_{\alpha \beta \mu}(x, y)=D^{\alpha} A_{\alpha \mu \nu}(x, y)=0$. As a consequence, also these fields have only transverse harmonics $a_{\mu \nu}^{N_{1}}(x)=a_{\mu}^{N_{7}}(x)=a^{N_{21}}(x)=0$. Substituting the resulting expansions into the $d=11$ field equations, the coefficients of each independent spherical harmonic yield the $d=4$ field equations.

In the Einstein equation for $R_{\mu \nu}$ only $Y^{N_{1}}$ spherical harmonics appear without derivatives. Thus there is only one field equation, i.e. one KK tower, for traceless symmetric tensors in $A d S_{4}$. 


\begin{tabular}{|l|l|l|l|l|l|l|}
\hline Spin & Field & $\mathrm{SO}(7)$ & $\mathrm{SO}(8)$ & $4(M L)^{2}$ & $\Delta$ & $\ell$ \\
\hline $2^{+}$ & $h_{(\mu \nu)}^{\prime}$ & $N_{1}$ & $(\ell, 0,0,0)$ & $\ell(\ell+6)$ & $\Delta=\frac{\ell}{2}+3$ & $\ell \geq 0$ \\
\hline $1_{1}^{-}$ & $h_{\mu \alpha}$ & $N_{7}$ & $(\ell, 1,0,0)$ & $\ell(\ell+2)$ & $\Delta=\frac{\ell}{2}+2$ & $\ell \geq 0$ \\
$1_{2}^{-}$ & $A_{\mu \nu \alpha}$ & $N_{7}$ & $(\ell-2,1,0,0)$ & $(\ell+6)(\ell+4)$ & $\Delta=\frac{\ell}{2}+4$ & $\ell \geq 2$ \\
\hline $1^{+}$ & $A_{\mu \alpha \beta}$ & $N_{21}$ & $(\ell-1,0,1,1)$ & $(\ell+2)(\ell+4)$ & $\Delta=\frac{\ell}{2}+3$ & $\ell \geq 1$ \\
\hline $0_{1}^{+}$ & $A_{\mu \nu \rho}$ & $N_{1}$ & $(\ell+2,0,0,0)^{*}$ & $(\ell+2)(\ell-4)$ & $\Delta=\frac{\ell}{2}+1$ & $\ell \geq 0$ \\
$0_{2}^{+}$ & $h_{\alpha \alpha}, h_{\lambda \lambda}^{\prime}$ & $N_{1}$ & $(\ell-2,0,0,0)$ & $(\ell+10)(\ell+4)$ & $\Delta=\frac{\ell}{2}+5$ & $\ell \geq 2$ \\
\hline $0_{3}^{+}$ & $h_{(\alpha \beta)}$ & $N_{27}$ & $(\ell-2,2,0,0)$ & $\ell(\ell+6)$ & $\Delta=\frac{\ell}{2}+3$ & $\ell \geq 2$ \\
\hline $0_{1}^{-}$ & $A_{\alpha \beta \gamma}$ & $N_{35}$ & $(\ell, 0,2,0)$ & $(\ell-2)(\ell+4)$ & $\Delta=\frac{\ell}{2}+2$ & $\ell \geq 0$ \\
\hline $0_{2}^{-}$ & $A_{\alpha \beta \gamma}$ & $N_{35}$ & $(\ell-2,0,0,2)$ & $(\ell+8)(\ell+2)$ & $\Delta=\frac{\ell}{2}+4$ & $\ell \geq 2$ \\
\hline
\end{tabular}

Table 1. Bosonic KK towers after compactification on $S^{7}$.

Examining the Einstein equation for $R_{\alpha \beta}$ one can see that the vector fields $B_{\mu}^{N_{7}}$ are massive and transversal, except for the lowest lying state corresponding to the Killing vectors on $S^{7}$. The spin-0 fields $\phi^{N_{27}}$ have a mass matrix $\Delta_{y}+12\left(\Delta_{y}\right.$ is the Hodge-de Rham operator). By a judicious gauge choice one can eliminate $H_{\mu}^{N_{1} \mu}$ in favour of $\pi^{N_{1}}$ namely $H_{\mu}^{N_{1} \mu}=\frac{9}{7} \pi^{N_{1}}$.

Collecting the coefficients of the spherical harmonics $Y_{\alpha}^{N_{7}}$ and $D_{\alpha} Y^{N_{1}}$ in the Einstein equation for $R_{\mu \alpha}$, one finds that the spin-1 spectrum consists of linear combinations of $B_{\mu}^{N_{7}}$ and $C_{\mu}^{N_{7}}$ (from $a_{\rho \sigma}^{N_{7}}$ ) and that one can eliminate the divergence $D^{\mu} H_{\mu \nu}^{N_{1}}$ in favour of $\pi^{N_{1}}$, $a_{\rho \sigma \tau}^{N_{1}}$ except when $Y^{N_{1}}$ is a constant.

Similarly, inspecting the equations for $p$-form field strengths $(p=1,2,3,4)$, one concludes that field expansions in spherical harmonics can be chosen such that only the first terms in the expansions survive with $Y$ s being transversal and traceless.

In particular, from the three-form field strength equation one finds that $a_{\mu \nu \rho}^{N_{1}}=$ $\varepsilon_{\mu \nu \rho \lambda} D^{\lambda} \sigma^{N_{1}}$. This implies that the divergence of $H_{\mu \nu}^{N_{1}}$ is proportional to a gradient.

From the four-form field strength equation one gets an equation for $\square_{x} \sigma^{N_{1}}$. Taking the trace of the equations for $R_{\mu \nu}$ and $R_{\alpha \beta}$, an equation involving $\square_{x} \sigma^{N_{1}}$ and $\square_{x} H_{\mu}^{N_{1} \mu}$ arises. Resolving the mixing between $a_{\mu \nu \rho}^{N_{1}}$ and $H_{\mu}^{N_{1} \mu}$ produces to independent combinations and as many KK towers of scalars.

From the two-form field strength equation one finds $D^{\mu} a_{\mu \nu}^{N_{7}}=0$, which implies $a_{\mu \nu}^{N_{7}}=$ $\varepsilon_{\mu \nu}^{\rho \sigma} D_{\rho} C_{\sigma}^{N_{7}}$. Using one of the three-form field strength equations one finds that $C_{\mu}^{N_{7}}$ and $B_{\mu}^{N_{7}}$ mix. Resolving the mixing one finds two KK towers, one of which starts with a massless vector corresponding to the internal Killing vectors of $S^{7}$.

After diagonalizing the bosonic field equations one obtains the mass spectrum summarized in table 1 . The resulting bosonic spectrum includes the massless graviton, 28 massless vectors of $\mathrm{SO}(8)$, corresponding to a combination of $B_{\mu}$ (in $h_{\mu \alpha}$ ) and $C_{\mu}$ (in 


\begin{tabular}{|l|l|l|l|l|}
\hline Spin & $\mathrm{SO}(8)$ & $4(M L)^{2}$ & $\Delta$ & $\ell$ \\
\hline$\left(\frac{3}{2}\right)_{1}$ & $(\ell, 0,0,1)$ & $(\ell+2)^{2}$ & $\Delta=\frac{\ell}{2}+\frac{5}{2}$ & $\ell \geq 0$ \\
\hline$\left(\frac{3}{2}\right)_{2}$ & $(\ell-1,0,1,0)$ & $(\ell+4)^{2}$ & $\Delta=\frac{\ell}{2}+\frac{7}{2}$ & $\ell \geq 1$ \\
\hline$\left(\frac{1}{2}\right)_{1}$ & $(\ell+1,0,1,0)^{*}$ & $\ell^{2}$ & $\Delta=\frac{\ell}{2}+\frac{3}{2}$ & $\ell \geq 0$ \\
\hline$\left(\frac{1}{2}\right)_{2}$ & $(\ell-1,1,1,0)$ & $(\ell+2)^{2}$ & $\Delta=\frac{\ell}{2}+\frac{5}{2}$ & $\ell \geq 1$ \\
\hline$\left(\frac{1}{2}\right)_{3}$ & $(\ell-2,1,0,1)$ & $(\ell+4)^{2}$ & $\Delta=\frac{\ell}{2}+\frac{7}{2}$ & $\ell \geq 2$ \\
\hline$\left(\frac{1}{2}\right)_{4}$ & $(\ell-2,0,0,1)$ & $(\ell+6)^{2}$ & $\Delta=\frac{\ell}{2}+\frac{9}{2}$ & $\ell \geq 2$ \\
\hline
\end{tabular}

Table 2. Fermionic KK towers after compactification on $S^{7}$.

$\left.A_{\mu \nu \alpha}\right), \mathbf{3 5}_{v}$ scalars $(\Delta=1)$ and $\mathbf{3 5}_{s}(\Delta=2)$ pseudoscalars with $\left(M L_{\mathrm{AdS}}\right)^{2}=-2$. In the supergravity literature $[19,29,30]$ masses of scalars are often shifted by $-R / 6$ so that $\left(M L_{\mathrm{AdS}}\right)^{2} \rightarrow\left(\tilde{M} L_{\mathrm{AdS}}\right)^{2}=\left(M L_{\mathrm{AdS}}\right)^{2}+2$. The 70 (pseudo)scalars in the $\mathcal{N}=8$ supergravity multiplet are 'massless' in the sense that $\left(\tilde{M} L_{\text {AdS }}\right)^{2}=0$. Moreover, there are three families of scalars and two families of pseudoscalar excitations. Three of them $\left(0_{2}^{+}, 0_{3}^{+}\right.$and $\left.0_{2}^{-}\right)$contain only states with positive mass square and correspond to irrelevant operators in the dual CFT. The remaining families $0_{1}^{+}$and $0_{1}^{-}$contain states with positive, zero and negative mass squared corresponding to irrelevant, marginal and relevant operators, respectively.

A similar analysis can be performed for fermionic fluctuations. In table 2 we summarize the fermionic mass spectrum.

The KK spectrum does not include the states with $*$ for $\ell=-1$, since they do not propagate in the bulk but live on the conformal boundary of $A d S_{4}$. They correspond to the singleton representation of $O s p(8 \mid 4)$ that consists of $8_{v}$ bosons $X^{i}$ with $\Delta=\frac{1}{2}$, $(M L)^{2}=-\frac{5}{4}$ and $8_{c}$ fermions $\psi^{\dot{a}}$ with $\Delta=1, M L=\frac{1}{2}$, both at the unitary bound.

The KK excitations on $S^{7}$ can be put in one-to-one correspondence with 'gaugeinvariant' composite operators on the boundary. The dictionary for bosonic operators schematically reads:

$$
\begin{aligned}
s=2^{+} & T_{\mu \nu, \Delta=\frac{\ell}{2}+3}^{i_{1} \ldots i_{\ell}}=\left(\partial_{\mu} X_{i} \partial_{\nu} X^{i}+\bar{\psi} \gamma_{\mu} \partial_{\nu} \psi\right) X^{i_{1}} \ldots X^{i_{\ell}} \\
s=1_{1}^{-} & J_{\mu, \Delta=\frac{\ell}{2}+2}^{[i j] i_{1} \ldots i_{\ell}}=\left(X^{[i} \partial_{\mu} X^{j]}+\bar{\psi} \Gamma^{i j} \gamma_{\mu} \psi\right) X^{i_{1}} \ldots X^{i_{\ell}} \\
s=1_{2}^{-} & J_{\mu, \Delta=\frac{\ell}{2}+4}^{[i j] i_{1} \ldots i_{\ell-2}}=\partial_{\mu} X_{i} \partial_{\nu} X^{i} \bar{\psi} \gamma^{\nu} \Gamma^{i j} \psi X^{i_{1}} \ldots X^{i_{\ell-2}} \\
s=1^{+} & J_{\mu, \Delta=\frac{\ell}{2}+3}^{a b i_{1} \ldots i_{\ell-1}}=\bar{\psi} \Gamma_{j k} \partial_{\mu} \psi\left(X_{i} \Gamma^{i j k}\right)^{a \dot{b}} X^{i_{1}} \ldots X^{i_{\ell-1}} \\
s=0_{1}^{+} & \Phi_{\Delta=\frac{\ell}{2}+1}^{i j i_{1} \ldots i_{\ell}}=X^{i} X^{j} X^{i_{1}} \ldots X^{i_{\ell}} \\
s=0_{2}^{+} & \Phi_{\Delta=\frac{\ell}{2}+5}^{i_{1} \ldots i_{\ell-2}}=\partial_{\mu} X^{i} \partial_{\nu} X_{i} \bar{\psi} \gamma^{\mu} \partial^{\nu} \psi X^{i_{1}} \ldots X^{i_{\ell-2}} \\
s=0_{3}^{+} & \Phi_{\Delta=\frac{\ell}{2}+3}^{[i j][k l] i_{1} \ldots i_{\ell-2}}=\left(\bar{\psi} \Gamma^{i j} \gamma_{\mu} \psi X^{[k} \partial^{\mu} X^{l]}\right) X^{i_{1}} \ldots X^{i_{\ell-2}}
\end{aligned}
$$




$$
\begin{aligned}
s & =0_{1}^{-} \quad \Phi_{\Delta=\frac{\ell}{2}+2}^{(\dot{a} \dot{b}) i_{1} \ldots i_{\ell}}=\bar{\psi}^{\dot{a}} \psi^{\dot{b}} X^{i_{1}} \ldots X^{i_{\ell}} \\
s & =0_{2}^{-} \quad \Phi_{\Delta=\frac{\ell}{2}+4}^{(a b) i_{1} \ldots i_{\ell-2}}=\left(\Gamma^{i j k l}\right)^{a b} X_{i} \partial^{\mu} X_{j} \bar{\psi} \Gamma_{k l} \partial_{\mu} \psi X^{i_{1}} \ldots X^{i_{\ell-2}}
\end{aligned}
$$

A similar dictionary can be compiled for fermions.

\section{Polynomial representations for $\mathrm{SO}(8)$ and $\mathrm{U}(4)$}

In order to decompose KK harmonics on $S^{7}=\mathrm{SO}(8) / \mathrm{SO}(7)$ into KK harmonics on $\mathbb{C P}^{3}=$ $\mathrm{U}(4) / \mathrm{U}(3) \times \mathrm{U}(1)$, we will present the construction of arbitrary representations of $\mathrm{SO}(8)$ in the space of polynomials of 12 variables. The latter are the coordinates of the subgroup $Z_{+}^{\mathrm{SO}(8)}$ generated by the raising operators of $\mathrm{SO}(8)$. We will then describe a technique which allows to identify which of the above polynomials correspond to highest weight states of representations of $\mathrm{U}(4) \subset \mathrm{SO}(8)$. The method we use is quite standard in representation theory of Lie groups (see e.g. chapter 16 of [31]).

It is convenient to start with $\mathrm{SO}(8, \mathbb{C})$ defined as the group of $8 \times 8$ complex matrices which leave invariant the quadratic form $X^{T} C^{(8)} X$, where $X$ is a complex (column) vector whose components will be enumerated as $X^{1}, X^{2}, X^{3}, X^{4}, X^{\tilde{4}}, X^{\tilde{3}}, X^{\tilde{2}}, X^{\tilde{1}}$ and $C^{(8)}$ is an $8 \times 8$ matrix with 1's on SW-NE (anti)diagonal:

$$
C_{i j}^{(8)}=C_{\tilde{i} j}^{(8)}=0, \quad C_{i \tilde{j}}^{(8)}=C_{\tilde{j} i}^{(8)}=\delta_{i j}, \quad i, j=1,2,3,4
$$

By definition all matrices $g \in \mathrm{SO}(8)$ satisfy the condition $g^{T} C^{(8)} g=C^{(8)}$. Eventually, in order to select the compact real form $\mathrm{SO}(8)$ of our interest, one should identify the coordinates $X^{\tilde{i}}$ with $\bar{X}^{i}$ (bar means complex conjugate). A generic $\mathrm{SO}(8)$ matrix $g$ can be (uniquely) decomposed as (Gauss decomposition):

$$
g=\zeta \lambda z
$$

where $\zeta \in Z_{-}, z \in Z_{+}, \lambda \in \Lambda$ with $Z_{+}\left(Z_{-}\right)$being the subgroup of lower (upper) triangular matrices with 1's on the diagonal and $\Lambda$ is the subgroup of diagonal matrices (Cartan subgroup). Let's set $\lambda=\operatorname{Diag}\left(\lambda_{1}, \lambda_{2}, \lambda_{3}, \lambda_{4}, \lambda_{4}^{-1}, \lambda_{3}^{-1}, \lambda_{2}^{-1}, \lambda_{1}^{-1}\right)$. We will realize the irreducible representations of the group $\mathrm{SO}(8)$ on some spaces of functions defined on it. In particular, the role of the highest weight vector will be played by the function:

$$
\alpha(g)=\lambda_{1}^{m_{1}} \lambda_{2}^{m_{2}} \lambda_{3}^{m_{3}} \lambda_{4}^{m_{4}}
$$

where $m_{1} \geq m_{2} \geq m_{3} \geq\left|m_{4}\right|$ ( $m_{i}$ are either all integers or all half-integers) uniquely characterize the irrep. The eigenvalues $\lambda_{i}$ can be expressed in terms of the matrix elements of $g$ explicitly:

$$
\lambda_{p}=\frac{\Delta_{p}}{\Delta_{p-1}}, \quad p=1,2,3,4
$$


where $\Delta_{0}=1$ and $\Delta_{p}, p=1,2,3,4$ are the diagonal minors

$$
\Delta_{p}=\left|\begin{array}{ccc}
g_{11} & \cdots & g_{1 p} \\
\vdots & \cdots & \vdots \\
g_{p 1} & \cdots & g_{p p}
\end{array}\right|
$$

Introducing the notation $S_{-}=\frac{\Delta_{3}}{\sqrt{\Delta_{4}}}, S_{+}=\sqrt{\Delta_{4}}$ (it is easy to see that $S_{+,-}$polynomially depend on the matrix elements of $g$ ) we can rewrite eq. (4.3) as

$$
\alpha(g)=\Delta_{1}^{\ell_{1}} \Delta_{2}^{\ell_{2}} S_{-}^{\ell_{3}} S_{+}^{\ell_{4}}
$$

where $\ell_{1}=m_{1}-m_{2}, \ell_{2}=m_{2}-m_{3}, \ell_{3}=m_{3}-m_{4}$ and $\ell_{4}=m_{3}+m_{4}$ are non-negative integers commonly referred as the Dynkin labels of the irrep. Consider the space $\mathcal{R}_{\alpha}$ of all linear combinations of the functions $\alpha\left(g g_{0}\right), g_{0} \in \mathrm{SO}(8)$. $\mathrm{SO}(8)$ is represented in $\mathcal{R}_{\alpha}$ simply by the right multiplication of the argument. As already mentioned the function $\alpha(g)$ plays the role of the highest weight state. For any function $f(g) \in \mathcal{R}_{\alpha}$ we have $f(\zeta \lambda z)=\alpha(\lambda) f(z)$ which shows that to restore its full $g$-dependence it is sufficient to only know the values the function assumes on the subgroup $Z_{+}$. This is why actually we get representation on a space of functions of $z$, in fact polynomials due to the polynomial dependence on $g$ of $\alpha(g)$ mentioned earlier.

There is an elegant way to characterize this space of polynomials. Consider the four raising generators corresponding to the simple roots

$$
\begin{aligned}
e_{1}=E_{12}-E_{\tilde{2} \tilde{1}} ; & e_{2}=E_{23}-E_{\tilde{3} \tilde{2}} \\
e_{-}=E_{34}-E_{\tilde{3} \tilde{4}} ; & e_{+}=E_{3 \tilde{4}}-E_{\tilde{4} 3}
\end{aligned}
$$

where $E_{p q}$ denotes the $8 \times 8$ matrix whose only non-zero entry 1 is at the position $(p, q)$. Denote their left action on $\mathcal{R}_{\alpha}$ by $\mathcal{D}_{1}, \mathcal{D}_{2}, \mathcal{D}_{-}, \mathcal{D}_{+}$. It is not difficult to prove that

$$
\begin{aligned}
& \mathcal{D}_{1}^{\ell_{1}+1} \alpha(g)=0 \\
& \mathcal{D}_{2}^{\ell_{2}+1} \alpha(g)=0 \\
& \mathcal{D}_{-}^{\ell_{3}+1} \alpha(g)=0 \\
& \mathcal{D}_{+}^{\ell_{4}+1} \alpha(g)=0 .
\end{aligned}
$$

The key observation is that the same equations are valid also for arbitrary functions $f \in \mathcal{R}_{\alpha}$, since they are all generated by $\alpha(g)$ through right multiplications which commute with left multiplications. Below we will use a convenient explicit parametrization of $Z_{+} \subset \mathrm{SO}(8)$ in terms of two $4 \times 4$ matrices $\eta$ and $a$

$$
\eta=\left(\begin{array}{cccc}
1 & \eta_{12} & \eta_{13} & \eta_{14} \\
0 & 1 & \eta_{23} & \eta_{24} \\
0 & 0 & 1 & \eta_{34} \\
0 & 0 & 0 & 1
\end{array}\right) ; \quad a=\left(\begin{array}{cccc}
a_{14} & a_{13} & a_{12} & 0 \\
a_{24} & a_{23} & 0 & -a_{12} \\
a_{34} & 0 & -a_{23} & -a_{13} \\
0 & -a_{34} & -a_{24} & -a_{14}
\end{array}\right)
$$


Let us further introduce the $8 \times 8$ matrices which in $2 \times 2$ block form read

$$
z_{0}=\left(\begin{array}{cc}
\eta & 0 \\
0 & \tilde{\eta}
\end{array}\right) ; \quad z^{\prime}=\left(\begin{array}{cc}
1 & a \\
0 & 1
\end{array}\right),
$$

where

$$
\tilde{\eta}=\left(\begin{array}{cccc}
1 & -\eta_{34} & -\eta_{24}+\eta_{23} \eta_{34}-\eta_{14}+\eta_{12} \eta_{24}+\eta_{13} \eta_{34}-\eta_{12} \eta_{23} \eta_{34} \\
0 & 1 & -\eta_{23} & -\eta_{13}+\eta_{12} \eta_{23} \\
0 & 0 & 1 & -\eta_{12} \\
0 & 0 & 0 & 1
\end{array}\right)
$$

An arbitrary $z \in Z_{+}$can be (uniquely) represented as

$$
z=z^{\prime} z_{0}
$$

Left multiplication by raising generators (4.7) induces infinitesimal motion on the parameters $a, \eta$. A straightforward algebra shows that e.g.

$$
\left(1+\epsilon e_{1}\right) z(a, \eta)=z(a+\delta a, \eta+\delta \eta)+O\left(\epsilon^{2}\right)
$$

where the non-trivial variations are

$$
\delta \eta_{12}=\epsilon, \delta \eta_{13}=\epsilon \eta_{23}, \delta \eta_{14}=\epsilon \eta_{24}, \delta a_{13}=\epsilon a_{23}, \delta a_{14}=\epsilon a_{24} .
$$

Similarly examining the remaining three generators we find

$$
\begin{aligned}
& \mathcal{D}_{1}=\partial_{\eta_{12}}+\eta_{23} \partial_{\eta_{13}}+a_{23} \partial_{a_{13}}+a_{24} \partial_{a_{14}} \\
& \mathcal{D}_{2}=\partial_{\eta_{23}}+\eta_{34} \partial_{\eta_{24}}+a_{13} \partial_{a_{12}}+a_{34} \partial_{a_{24}} \\
& \mathcal{D}_{-}=\partial_{\eta_{34}}+a_{14} \partial_{a_{13}}+a_{24} \partial_{a_{23}} \\
& \mathcal{D}_{+}=\partial_{a_{34}} .
\end{aligned}
$$

Thus any irreducible representation of $\mathrm{SO}(8)$ is realized on the space of polynomials of 12 variables $a, \eta$ subject to the constraints

$$
\begin{aligned}
\left(\partial_{\eta_{12}}+\eta_{23} \partial_{\eta_{13}}+a_{23} \partial_{a_{13}}+a_{24} \partial_{a_{14}}\right)^{\ell_{1}+1} f(a, \eta) & =0 \\
\left(\partial_{\eta_{23}}+\eta_{34} \partial_{\eta_{24}}+a_{13} \partial_{a_{12}}+a_{34} \partial_{a_{24}}\right)^{\ell_{2}+1} f(a, \eta) & =0 \\
\left(\partial_{\eta_{34}}+a_{14} \partial_{a_{13}}+a_{24} \partial_{a_{23}}\right)^{\ell_{3}+1} f(a, \eta) & =0 \\
\left(\partial_{a_{34}}\right)^{\ell_{4}+1} f(a, \eta) & =0 .
\end{aligned}
$$

Note that the constant polynomial always satisfies (4.15) and corresponds to the highest weight state. Considering right multiplication it is not difficult to find explicit expressions for the generators of $\mathrm{SO}(8)$ as operators acting on the space of polynomials. For our later proposes let us specify how the diagonal part $\Lambda \subset \mathrm{SO}(8)$ is represented. Since

$$
z(a, \eta) \lambda=\lambda \lambda^{-1} z(a, \eta) \lambda=\lambda z\left(a^{\prime}, \eta^{\prime}\right)
$$


where

$$
a_{i j}^{\prime}=\lambda_{j}^{-1} \lambda_{i}^{-1} a_{i j} ; \quad \eta_{i j}^{\prime}=\lambda_{j} \lambda_{i}^{-1} \eta_{i j}
$$

we simply get

$$
\lambda \circ f(a, \eta)=\lambda_{1}^{m_{1}} \lambda_{2}^{m_{2}} \lambda_{3}^{m_{3}} \lambda_{4}^{m_{4}} f\left(a^{\prime}, \eta^{\prime}\right)
$$

Notice that the variable $a_{i j}$ shifts the weights as $m_{i} \rightarrow m_{i}-1, m_{j} \rightarrow m_{j}-1$ while the variable $\eta_{i j}$ shifts them as $m_{i} \rightarrow m_{i}-1, m_{j} \rightarrow m_{j}+1$.

Consider now the $G L(4, C) \subset \mathrm{SO}(8, C)$ subgroup whose off-diagonal blocks in $2 \times 2$ block notation are zero. This subgroup does not mix the coordinates $X^{i}$ with $X^{\tilde{i}}$ and after restriction to the real sector it becomes the subgroup $\mathrm{U}(4) \subset \mathrm{SO}(8)$.

In other words, for the reduction from $S^{7}$ to $S^{7} / \mathbb{Z}_{k}$ or $\mathbb{C P}^{3} \ltimes S^{1}$ we are interested in, the decomposition $\mathrm{SO}(8) \rightarrow \mathrm{SO}(6) \times \mathrm{SO}(2)$ is given by the embedding

$$
\mathbf{8}_{\mathbf{v}}(1,0,0,0) \rightarrow \mathbf{4}_{+1}[0,1,0]+\mathbf{4}_{-1}^{*}[0,0,1]
$$

where $\left(\ell_{1}, \ell_{2}, \ell_{3}, \ell_{4}\right)$ and $[k, l, m]$ denote $\mathrm{SO}(8)$ and $\mathrm{SO}(6)$ Dynkin labels respectively. As a result, for the Adjoint representation one has

$$
\mathbf{2 8}(0,1,0,0) \rightarrow \mathbf{1 5}_{0}[0,1,1]+\mathbf{1}_{0}[0,0,0]+\mathbf{6}_{+2}[1,0,0]+\mathbf{6}_{-2}[1,0,0]
$$

while

$$
\begin{aligned}
& \mathbf{8}_{\mathbf{s}}(0,0,0,1) \rightarrow \mathbf{6}_{0}[1,0,0]+\mathbf{1}_{+2}[0,0,0]+\mathbf{1}_{-2}[0,0,0] \\
& \mathbf{8}_{\mathbf{c}}(0,0,1,0) \rightarrow \mathbf{4}_{-1}[0,1,0]+\mathbf{4}_{+1}^{*}[0,0,1]
\end{aligned}
$$

for the spinorial representations.

Our goal is to identify the highest weight states of this subgroup inside the space of polynomials of a given representation of $\mathrm{SO}(8)$. It is evident from the decomposition (4.12), (4.11) that the right action by the raising operators of $G L(4)$ subgroup $e_{1}, e_{2}$, $e_{-}$(see eq. (4.7)) shifts the parameters $\eta$ and leave the parameters $a$ untouched. Thus, in order to be a highest weight state, a polynomial, besides satisfying the equations (4.15) should be independent of $\eta$. The indicator system for the highest weight states becomes

$$
\begin{aligned}
\left(a_{23} \partial_{a_{13}}+a_{24} \partial_{a_{14}}\right)^{\ell_{1}+1} f(a) & =0 \\
\left(a_{13} \partial_{a_{12}}+a_{34} \partial_{a_{24}}\right)^{\ell_{2}+1} f(a) & =0 \\
\left(a_{14} \partial_{a_{13}}+a_{24} \partial_{a_{23}}\right)^{\ell_{3}+1} f(a) & =0 \\
\left(\partial_{a_{34}}\right)^{\ell_{4}+1} f(a) & =0 .
\end{aligned}
$$

Solving these equations one can fully decompose KK harmonics on $S^{7}$ into KK harmonics of $\mathbb{C P}^{3} \times S^{1}$ which is our next task. 


\section{From $S^{7}$ to $\mathbb{C P}^{3} \ltimes S^{1}$}

$S^{7}$ is a $\mathrm{U}(1)$ bundle over $\mathbb{C P}^{3}$. The $\mathbb{C P}^{3}$ solution of the $d=10$ theory can be obtained from the $S^{7}$ solution of the $d=11$ theory by Hopf fibration, i.e. keeping only $\mathrm{U}(1)$ invariant states [26]. The compactification on $\mathbb{C P}^{3}$ of the $d=10$ theory yields a four dimensional theory with $\mathcal{N}=6$ supersymmetry and with gauge group $\mathrm{SO}(6) \times \mathrm{SO}(2)$.

The truncation from $S^{7}$ to $\mathbb{C P}^{3} \ltimes S^{1}$ cannot be thought of as spontaneous (super)symmetry breaking and one has to really discard the states that are projected out by $\mathbb{Z}_{k}$ or $\mathrm{SO}(2)$ for $k \rightarrow \infty$ even if it acts freely. In particular we will later check that no Higgsing can account for the breaking of $\mathrm{SO}(8)$ to $\mathrm{SO}(6) \times \mathrm{SO}(2)$ but rather the coset vectors are dressed with monopole operators and become massive for $k \neq 1,2[7,8,22,28,32]$.

Let us start with the KK towers of bosons. Using the procedure described in the previous section or otherwise, for scalar spherical harmonics with Dynkin labels $(\ell, 0,0,0)$ one finds as independent polynomials $\left\{a_{14}^{m} \mid m=0, \ldots, \ell\right\}$. Thus the following decomposition holds:

$$
N_{1}: \quad(\ell, 0,0,0) \rightarrow \oplus[0, \ell-m, m]_{\ell-2 m}
$$

where the subscript is the $\mathrm{SO}(2)$ charge $Q$ of the appropriate representation.

For vector spherical harmonics with $\mathrm{SO}(8)$ Dynkin labels $(\ell-2,1,0,0)$ one gets $\left\{a_{12} a_{14}^{m}, a_{24} a_{14}^{m},\left(a_{13} a_{24}-a_{14} a_{23}\right) a_{14}^{m}, a_{14}^{m} \mid m=0, \ldots, \ell\right\}$ as independent polynomials. The $\mathrm{SO}(8)$ representation decomposes into $\mathrm{SO}(6)$ representations as:

$$
\begin{aligned}
N_{7}: \quad(\ell, 1,0,0) \rightarrow & \oplus[0, \ell-m, m]_{\ell-2 m} \oplus[0, \ell-m+1, m+1]_{\ell-2 m} \\
& \oplus[1, \ell-m, m]_{\ell-2 m-2} \oplus[1, \ell-m, m]_{\ell-2 m+2}
\end{aligned}
$$

One obtains the decomposition of the representation $(\ell-2,1,0,0)$ from the previous one by shifting $\ell$ to $\ell-2$. In what follows we will simply omit the decompositions which differ by shifts of the parameter $\ell$.

For two-form spherical harmonics with $\mathrm{SO}(8)$ Dynkin labels $(\ell-1,0,1,1)$ one finds $\left\{a_{14}^{m}, a_{23} a_{14}^{m}, a_{34} a_{14}^{m}, a_{23} a_{34} a_{14}^{m},\left(a_{34} a_{12}-a_{13} a_{24}\right) a_{14}^{m}, a_{23}\left(a_{23} a_{14}+a_{34} a_{12}-\right.\right.$ $\left.\left.\left.a_{13} a_{24}\right) a_{14}^{m}\right), a_{13} a_{14}^{n}, a_{34} a_{13} a_{14}^{n},\left(a_{34} a_{12}-a_{13} a_{24}\right) a_{13} a_{14}^{n} \mid m=0, \ldots, \ell-1, n=0, \ldots, \ell-2\right\}$ as independent polynomials. One then finds the following decomposition:

$$
\begin{aligned}
N_{21}: \quad(\ell-1,0,1,1) \rightarrow & \oplus[0, \ell-m, m]_{\ell-2 m-4} \oplus[0, \ell-m-1, m+1]_{\ell-2 m+2} \\
& \oplus[1, \ell-m, m]_{\ell-2 m-2} \oplus[1, \ell-m-1, m+1]_{\ell-2 m} \\
& \oplus[0, \ell-m, m]_{\ell-2 m} \oplus[0, \ell-m-1, m+1]_{\ell-2 m-2} \\
& \oplus[1, \ell-n-2, n]_{\ell-2 n-4} \oplus[2, \ell-n-2, n]_{\ell-2 n-2} \\
& \oplus[1, \ell-n-2, n]_{\ell-2 n}
\end{aligned}
$$

The decomposition of the KK towers corresponding to $0_{1}^{+}$and $0_{2}^{+}$can be found from the decomposition of $2^{+}$via appropriate shifts. 
For second rank symmetric traceless harmonics with Dynkin labels $(\ell-2,2,0,0)$ the polynomials are: $\left\{a_{14}^{m}, a_{12} a_{14}^{m}, a_{12}\left(a_{23} a_{14}-a_{13} a_{24}\right) a_{14}^{m}, a_{12}^{2} a_{14}^{m}, a_{12} a_{24} a_{14}^{m}, a_{24} a_{14}^{m}, a_{24}\left(a_{23} a_{14}-\right.\right.$ $\left.\left.a_{13} a_{24}\right) a_{14}^{m},\left(a_{13} a_{24}-a_{14} a_{23}\right) a_{14}^{m},\left(a_{14} a_{23}-a_{13} a_{24}\right)^{2} a_{14}^{m}, a_{24}^{2} a_{14}^{m}, \mid m=0, \ldots, \ell-2\right\}$. The SO(6) representations decomposed from $\mathrm{SO}(8)$ 's are:

$$
\begin{aligned}
N_{27}: \quad(\ell-2,2,0,0) \rightarrow & \oplus[2, \ell-m-2, m]_{\ell-2 m+2} \oplus[1, \ell-m-2, m]_{\ell-2 m} \\
& \oplus[1, \ell-m-2, m]_{\ell-2 m-4} \oplus[0, \ell-m-1, m+1]_{\ell-2 m-2} \\
& \oplus[0, \ell-m-2, m]_{\ell-2 m-2} \oplus[1, \ell-m-1, m+1]_{\ell-2 m} \\
& \oplus[1, \ell-m-1, m+1]_{\ell-2 m-4} \oplus[2, \ell-m-2, m]_{\ell-2 m-2} \\
& \oplus[2, \ell-m-2, m]_{\ell-2 m-6} \oplus[0, \ell-m, m+2]_{q=\ell-2 m-2}
\end{aligned}
$$

For the three-form spherical harmonic with $\mathrm{SO}(8)$ Dynkin labels $(\ell, 0,2,0)$ one finds $\left\{\left(a_{14}^{m}+a_{23} a_{14}^{m}+a_{23}^{2} a_{14}^{m}\right), a_{13}\left(a_{14}^{n}+a_{23} a_{14}^{n}\right), a_{13}^{2} a_{14}^{p} \mid m=0, \ldots, \ell, n=0, \ldots, \ell-1, p=\right.$ $0, \ldots, \ell-2\}$ polynomials. The representation $(\ell, 0,2,0)$ decomposes as:

$$
\begin{aligned}
N_{35}: \quad(\ell, 0,2,0) \rightarrow & \oplus[0, \ell-m, m+2]_{\ell-2 m+2} \oplus[0, \ell-m+1, m+1]_{\ell-2 m} \\
& \oplus[0, \ell-m+2, m]_{\ell-2 m-2} \oplus[1, \ell-n-1, n+1]_{\ell-2 n} \\
& \oplus[1, \ell-n, n]_{\ell-2 n-2} \oplus[2, \ell-p-2, p]_{\ell-2 p-2}
\end{aligned}
$$

For the three-form spherical harmonic with $\mathrm{SO}(8)$ Dynkin labels $(\ell-2,0,0,2)$ one has $\left\{\left(a_{14}^{m},\left(a_{14} a_{23}+a_{12} a_{34}-a_{13} a_{24}\right) a_{14}^{m},\left(a_{12} a_{34}-a_{13} a_{24}+a_{14} a_{23}\right)^{2} a_{14}^{m}, a_{34} a_{14}^{m}, a_{34}\left(a_{24} a_{13}-\right.\right.\right.$ $\left.\left.a_{34} a_{12}-a_{14} a_{23}\right) a_{14}^{m}, a_{34}^{2} a_{14}^{m} \mid m=0, \ldots, \ell-2\right)$ and the following decomposition:

$$
\begin{aligned}
N_{35}^{\prime}: \quad(\ell-2,0,0,2) \rightarrow & \oplus[0, \ell-m-2, m]_{\ell-2 m-2} \oplus[0, \ell-m-2, m]_{\ell-2 m+2} \\
& \oplus[1, \ell-m-2, m]_{\ell-2 m-4} \oplus[1, \ell-m-2, m]_{\ell-2 m} \\
& \oplus[2, \ell-m-2, m]_{\ell-2 m-2} \oplus[0, \ell-m-2, m]_{\ell-2 m-6}
\end{aligned}
$$

Let us now consider the fermionic KK towers. There are two gravitini in the $\mathrm{SO}(8)$ representations $(\ell, 0,0,1)$ and $(\ell-1,0,1,0)$.

For the $\mathrm{SO}(8)$ representation $(\ell, 0,0,1)$ one finds $\left\{a_{14}^{m},\left(a_{14} a_{23}+a_{12} a_{34}-\right.\right.$ $\left.\left.a_{13} a_{24}\right) a_{14}^{m}, a_{34} a_{14}^{m} \mid m=0, \ldots, \ell\right\}$ as polynomials and the following decomposition holds

$$
(\ell, 0,0,1) \rightarrow \oplus[0, \ell-m, m]_{\ell-2 m+2} \oplus[0, \ell-m, m]_{\ell-2 m-2} \oplus[1, \ell-m, m]_{\ell-2 m}
$$

For the $\mathrm{SO}(8)$ representation $(\ell-1,0,1,0)$ the independent polynomials are $\left\{a_{14}^{m}\right.$, $\left.a_{23} a_{14}^{m}, a_{13} a_{14}^{n} \mid m=0, \ldots, \ell-1, n=0, \ldots, \ell-2\right\}$ and is decomposed as:

$$
\begin{aligned}
(\ell-1,0,1,0) \rightarrow & \oplus[0, \ell-m-1, m+1]_{\ell-2 m} \oplus[0, \ell-m, m]_{\ell-2 m-2} \\
& \oplus[1, \ell-n-2, n]_{\ell-2 n-2}
\end{aligned}
$$

There are other fermions in the representations $(\ell+1,0,1,0),(\ell-2,0,0,1),(\ell-1,1,1,0)$ and $(\ell-2,1,0,1)$. 
For the $\mathrm{SO}(8)$ representation $(\ell-1,1,1,0)$ the polynomials have the form $\left\{a_{14}^{m}, a_{23} a_{14}^{m}, \quad a_{23}\left(a_{13} a_{24}-a_{14} a_{23}\right) a_{14}^{m}, a_{24} a_{14}^{m}, a_{13} a_{24} a_{14}^{m}, a_{23} a_{24} a_{14}^{m}, a_{12} a_{14}^{m}\right.$, $\left.a_{12} a_{23} a_{14}^{m}, a_{13} a_{14}^{n}, a_{13}\left(a_{13} a_{24}-a_{23} a_{14}\right) a_{14}^{n}, a_{12} a_{13} a_{14}^{n} \mid m=0, \ldots, \ell-1, n=0, \ldots, \ell-2\right\}$ and one has the following decomposition:

$$
\begin{aligned}
(\ell-1,1,1,0) \rightarrow & \oplus[1, \ell-m-1, m+1]_{\ell-2 m+2} \oplus[1, \ell-m, m]_{\ell-2 m} \\
& \oplus[1, \ell-m, m]_{\ell-2 m-4} \oplus[0, \ell-m, m+2]_{\ell-2 m} \\
& \oplus[1, \ell-m-1, m+1]_{\ell-2 m-2} \oplus[0, \ell-m+1, m+1]_{\ell-2 m-2} \\
& \oplus[0, \ell-m-1, m+1]_{\ell-2 m} \oplus[0, \ell-m, m]_{\ell-2 m-2} \\
& \oplus[2, \ell-n-2, n]_{\ell-2 n} \oplus[2, \ell-n-2, n]_{\ell-2 n-4} \\
& \oplus[1, \ell-n-2, n]_{\ell-2 n-2}
\end{aligned}
$$

Finally for the $\mathrm{SO}(8)$ representation $(\ell-2,1,0,1)$ the polynomials have the form $\left\{a_{14}^{m}\right.$, $\left(a_{14} a_{23}-a_{13} a_{24}\right) a_{14}^{m},\left(a_{13} a_{24}-a_{12} a_{34}-a_{14} a_{23}\right)\left(a_{14} a_{23}-a_{13} a_{24}\right) a_{14}^{m}, a_{12} a_{14}^{m}, a_{12}\left(a_{12} a_{34}-\right.$ $\left.a_{13} a_{24}+a_{14} a_{23}\right) a_{14}^{m}, a_{24} a_{14}^{m}, a_{24}\left(a_{12} a_{34}-a_{13} a_{24}+a_{14} a_{23}\right) a_{14}^{m}, a_{34} a_{14}^{m}, a_{34}\left(a_{13} a_{24}-\right.$ $\left.\left.a_{14} a_{23}\right) a_{14}^{m}, a_{34} a_{24} a_{14}^{m}, a_{34} a_{12} a_{14}^{m} \mid m=0, \ldots, \ell-2\right\}$ and the decomposition reads

$$
\begin{aligned}
(\ell-2,1,0,1) \rightarrow & \oplus[1, \ell-m-2, m]_{\ell-2 m+2} \oplus[1, \ell-m-2, m]_{\ell-2 m-2} \\
& \oplus[1, \ell-m-2, m]_{\ell-2 m-6} \oplus[0, \ell-m-2, m]_{\ell-2 m} \\
& \oplus[0, \ell-m-2, m]_{\ell-2 m-4} \oplus[0, \ell-m-1, m+1]_{\ell-2 m} \\
& \oplus[0, \ell-m-1, m+1]_{\ell-2 m-4} \oplus[2, \ell-m-2, m]_{\ell-2 m} \\
& \oplus[2, \ell-m-2, m]_{\ell-2 m-4} \oplus[1, \ell-m-1, m+1]_{\ell-2 m-2} \\
& \oplus[1, \ell-m-2, m]_{\ell-2 m-2}
\end{aligned}
$$

The relevant $\mathrm{SO}(8) \rightarrow \mathrm{SO}(6) \times \mathrm{SO}(2)$ decomposition is given by the embedding (4.19), (4.21), (4.22). In particular this implies

$$
\begin{aligned}
\mathbf{3 5}_{\mathbf{v}}(2,0,0,0) \rightarrow & \mathbf{1 5}_{0}[0,1,1]+\mathbf{1 0}_{+2}[0,2,0]+\mathbf{1 0}_{-2}[0,0,2] \\
\mathbf{3 5}_{\mathbf{c}}(0,0,2,0) \rightarrow & \mathbf{1 5}_{0}[0,1,1]+\mathbf{1 0}_{+2}^{*}[0,0,2]+\mathbf{1 0}_{-2}[0,2,0] \\
\mathbf{3 5}_{\mathbf{s}}(0,0,0,2) \rightarrow & \mathbf{2 0}_{0}^{\prime}[2,0,0]+\mathbf{6}_{+2}[1,0,0]+\mathbf{6}_{-2}[1,0,0]+ \\
& \mathbf{1}_{0}[0,0,0]+\mathbf{1}_{+4}[0,0,0]+\mathbf{1}_{-4}[0,0,0]
\end{aligned}
$$

that are necessary to analyze the spectrum of scalars.

The zero charge spectrum i.e. the states which constitute the KK spectrum of Type IIA supergravity on $\mathbb{C P}^{3}$ can be easily identified in the above decompositions. For completeness and comparison with the original literature [26], we collect the relevant formulae in an appendix.

\subsection{A closer look at the KK spectrum}

As already observed, the $\mathbb{Z}_{k}$ orbifold projection from $S^{7}$ to $S^{7} / \mathbb{Z}_{k} \approx \mathbb{C P}^{3} \ltimes S^{1}$ cannot be thought of as spontaneous (super)symmetry breaking. 'Untwisted' states that are projected 
out do not simply become 'massive' but are rather eliminated from the spectrum. In particular in the large $k$ limit only $\mathrm{SO}(2)$ singlets survive. It is amusing to observe that only states with $\ell$ even on $S^{7}$ give rise to neutral states. This suggests that the parent theory could be either a compactification on $S^{7}$ or on $\mathbb{R} P^{7}=S^{7} / \mathbb{Z}_{2}$. Indeed both lead to $\mathrm{SO}(8)$ gauged supergravity corresponding to the 'massless' multiplet

$$
\left\{g_{\mu \nu}, 8 \psi_{\mu}, 28 A_{\mu}, 56 \lambda, 35^{+}+35^{-} \varphi\right\}
$$

Massless scalars, corresponding to marginal operators with $\Delta=3$ on the boundary, only appear in higher KK multiplets, i.e. in the $\mathbf{8 4 0}^{\prime}=(2,0,0,2)$ and $\mathbf{1 3 8 6}=(6,0,0,0)$. None of these can play the role of Stückelberg field for the 12 coset vectors in the $\mathbf{6}_{+2}+\mathbf{6}_{-2}$ of $\mathrm{SO}(8) / \mathrm{SO}(6) \times \mathrm{SO}(2)$.

Indeed, using the group theory techniques described in section 4 or otherwise, the decomposition of $\mathbf{8 4 0}^{\prime}=(2,0,2,0)$ under $\mathrm{SO}(8) \rightarrow \mathrm{SO}(6) \times \mathrm{SO}(2)$ reads

$$
\begin{aligned}
\mathbf{8 4 0}_{\mathbf{v c}}(2,0,2,0) \rightarrow & \mathbf{8 4}_{+4}[0,2,2]+\mathbf{7 0}_{+2}[0,3,1]+\mathbf{7 0}_{+2}[0,1,3]+\mathbf{6 4}_{+2}[1,1,1] \\
& +\mathbf{8 4}_{0}[0,2,2]+\mathbf{4 5}_{0}[1,2,0]+\mathbf{4 5}_{0}[1,0,2] \\
& +\mathbf{3 5}_{0}[0,4,0]+\mathbf{3 5}_{0}[0,0,4]+\mathbf{2 0}_{0}^{\prime}[2,0,0] \\
& +\mathbf{8 4}_{-4}[0,2,2]+\mathbf{7 0}_{-2}[0,3,1]+\mathbf{7 0}_{-2}[0,1,3]+\mathbf{6 4}_{-2}[1,1,1]
\end{aligned}
$$

This means that the massless scalars in the $\mathbf{8 4 0}_{\mathbf{v c}}(2,0,2,0)$ cannot account for the 'needed' Stückelberg fields in the $\mathbf{6}_{+2}+\mathbf{6}_{-2}$. Yet one can recognize massless scalars neutral under $\mathrm{SO}(2)$ that survive in $k \rightarrow \infty$ limit and transform non-trivially under $\mathrm{SO}(6)$. Turning them on in the bulk, e.g. in domain-wall solutions, should trigger RG flows to theories with lower supersymmetry on the boundary.

The same applies to the other massless scalars in the $\mathbf{1 3 8 6}(6,0,0,0)$, the totally symmetric product of $6 \mathbf{8}_{\mathbf{v}} \rightarrow \mathbf{4}_{+1}+\mathbf{4}_{-1}^{*}$. The relevant decomposition reads

$$
\begin{aligned}
\mathbf{1 3 8 6}(6,0,0,0) \rightarrow & \mathbf{8 4}_{+6}[0,6,0]+\mathbf{1 8 9}_{+4}[0,5,1]+\mathbf{2 7 0}_{+2}[0,4,2] \\
& +\mathbf{3 0 0}_{0}[0,3,3] \\
& +\mathbf{8 4}_{-6}[0,0,6]+\mathbf{1 8 9}_{-4}[0,1,5]+\mathbf{2 7 0}_{-2}[0,2,4]
\end{aligned}
$$

Once again there are no $\mathbf{6}_{+2}+\mathbf{6}_{-2}$. In this case, 'neutral' fields appear in the $\mathbf{3 0 0}$ representation of $\mathrm{SO}(6)$.

In the KK spectrum, neutral (wrt to $\mathrm{SO}(2)$ ) singlets (of $\mathrm{SO}(6)$ ) appear in the decomposition of $\mathbf{3 5}_{s}$ parity odd scalars $0_{2}^{-}$with $M^{2} L_{\text {AdS }}^{2}=10$ that reads

$$
\begin{aligned}
\mathbf{3 5}_{\mathbf{s}}(0,0,0,2) \rightarrow & \mathbf{2 0}_{0}^{\prime}[2,0,0]+\boldsymbol{6}_{+2}[1,0,0]+\mathbf{6}_{-2}[1,0,0] \\
& +\mathbf{1}_{0}[0,0,0]+\mathbf{1}_{+4}[0,0,0]+\mathbf{1}_{-4}[0,0,0]
\end{aligned}
$$

They correspond to boundary operators with dimension $\Delta=5$. The only other neutral singlets arise from the $\mathrm{SO}(8)$ singlet parity even scalar with $M^{2} L_{\mathrm{AdS}}^{2}=18$, i.e. $\Delta=6$. 
Neither ones belongs in the supergravity multiplet. ${ }^{1}$ They correspond to the 'stabilized' complexified Kähler deformation $\mathcal{J}+i B$ and as such couple to the Type IIA world-sheet instanton recently identified in [33]. Indeed the bosonic action schematically reads $S_{w s i}=$ $\int \mathcal{J}+i B=L^{2} / \alpha^{\prime}$ since $B=0$ in the ABJM model, while $B=l / k$ with $l=1, \ldots, k-1$ for the ABJ model involving fractional M2-branes. Effects induced by world-sheet instantons in Type IIA on $\mathbb{C P}^{3}$ should be dual to the non-perturbative corrections discussed in [34]. It may be worth to observe that in 'ungauged' $\mathcal{N}=6$ supergravity, arising from freely acting asymmetric orbifolds of Type II superstrings on tori, world-sheet and other asymmetric brane instantons $[35,36]$ should correct $\mathcal{R}^{4}$ terms very much as in their parents with $\mathcal{N}=8$ local supersymmetry.

Other non-perturbative effects are induced by E5-brane instantons that should mediate the process of annihilation of $k$ D0-branes into $N$ D4-branes wrapping $C P^{2}[7,22]$. In order to determine the action of such an instanton it is worth recalling that the pseudoscalar mode $B_{2}=\beta(x) J_{2}(y)$ is eaten by the vector field $A_{\mu}^{H}=k A_{\mu}^{D 4}-N A_{\mu}^{D 0}$ that becomes massive. The complete E5-brane instanton action should be $S_{E 5}=L^{6} / g_{s}^{2}\left(\alpha^{\prime}\right)^{3}+i \beta$ that indeed shifts under $\mathrm{U}(1)_{H}$ gauge transformations and as such can compensate for the 'charge' violation in the above process as in similar cases with unoriented D-brane instantons [37].

\section{Singleton, partition functions and higher spins}

In this section, we would like to discuss the higher spin (HS) extension of $\mathcal{N}=6$ gauged supergravity. Higher spin extensions of various supergravity theories in $A d S_{4}$ have been studied in [38-40] but to the best of our knowledge the case of $\mathcal{N}=6$ has been overlooked.

Let us start by briefly recalling some basic features of higher spin theories in $A d S_{4} .{ }^{2}$ In the non supersymmetric case the HS algebra represents an extension of the conformal group $\mathrm{SO}(3,2)$ that admits two singleton representations $\mathcal{D}(1 / 2,0)$ (free boson) and $\mathcal{D}(1,1 / 2)$ (free fermion). The two labels denote conformal dimension $\Delta$ and spin $s$. Indeed the maximal compact subgroup of $\mathrm{SO}(3,2)$ is $\mathrm{SO}(3) \times \mathrm{SO}(2) \approx \mathrm{SU}(2) \times \mathrm{U}(1)$ while 'Lorentz' transformations and dilatations commute and generate $\mathrm{SO}(2,1) \times \mathrm{SO}(1,1) \subset \mathrm{SO}(3,2)$. We will continue and call $\Delta$ the dimension and $s$ or $j$ spin. In 'radial' quantization the 'Hamiltonian' $\mathcal{H}$ has eigenvalues $\Delta$.

For later use let us collect here the partition functions of the two singletons that take into account their conformal descendants i.e. non vanishing derivatives. For free bosons such that $\partial^{2} X=0$ one has

$$
\mathcal{Z}_{B}(q)=\operatorname{Tr} q^{2 \mathcal{H}}=\frac{q-q^{5}}{\left(1-q^{2}\right)^{3}}=\frac{q+q^{3}}{\left(1-q^{2}\right)^{2}}
$$

\footnotetext{
${ }^{1}$ After gauging $\mathrm{SO}(8)$, the 70 scalars give rise to $\mathbf{3 5}_{\mathbf{v}}(2,0,0,0)$ and $\mathbf{3 5}_{\mathbf{c}}(0,0,2,0)$ which in turn decompose into $\mathbf{3 5}_{\mathbf{v}}(2,0,0,0) \rightarrow \mathbf{1 5}_{0}[0,1,1]+\mathbf{1 0}_{+2}[0,2,0]+\mathbf{1 0}_{-2}{ }_{-2}[0,0,2]$ and $\mathbf{3 5}_{\mathbf{c}}(0,0,2,0) \rightarrow \mathbf{1 5}_{0}[0,1,1]+$ $\mathbf{1 0}_{+2}^{*}[0,0,2]+\mathbf{1 0}_{-2}[0,2,0]$.

${ }^{2}$ See e.g. [41-45] and references therein for recent reviews of both Vasiliev's and geometric approaches.
} 
For free fermions $\not \partial \Psi=0$ one has

$$
\mathcal{Z}_{F}(q)=\operatorname{Tr} q^{2 \mathcal{H}}=2 \frac{q^{2}-q^{4}}{\left(1-q^{2}\right)^{3}}=2 \frac{q^{2}}{\left(1-q^{2}\right)^{2}}
$$

Combining $n_{b}=8_{v}$ free bosons and $n_{f}=8_{c}$ free fermions one finds the singleton representation of $O s p(8 \mid 4) \supset \mathrm{SO}(8) \times \mathrm{SO}(3,2)$, whose Witten index reads

$$
\mathcal{Z}_{\square}(q)=\operatorname{Tr}(-)^{F} q^{2 \mathcal{H}}=8_{v} \mathcal{Z}_{B}(q)-8_{c} \mathcal{Z}_{F}(q)
$$

One can also keep track of the spin of the states in the spectrum by including a chemical potential $y=e^{i \alpha}\left(y^{J_{3}}=e^{i \alpha J_{3}}\right)$ and find

$$
\begin{aligned}
& \mathcal{Z}_{B}(q, \alpha)=\frac{q\left(1-q^{4}\right)}{\left(1-q^{2}\right)\left(1-e^{i \alpha} q^{2}\right)\left(1-e^{-i \alpha} q^{2}\right)}=\frac{q\left(1+q^{2}\right)}{\left(1-2 q^{2} \cos \alpha+q^{4}\right)} \\
& \mathcal{Z}_{F}(q, \alpha)=\frac{q^{2}\left(1-q^{2}\right) \chi_{\frac{1}{2}}(\alpha)}{\left(1-q^{2}\right)\left(1-2 q^{2} \cos \alpha+q^{4}\right)}
\end{aligned}
$$

where

$$
\chi_{\frac{1}{2}}(\alpha)=2 \cos \frac{\alpha}{2}=\operatorname{tr}_{1 / 2} e^{i \alpha J_{3}}
$$

is the character of the fundamental representation of the 'Lorentz' group $\mathrm{SU}(2)$.

Before switching to higher spins, notice that $\mathbb{Z}_{k}$ acts on the singleton simply as

$$
8_{v} \rightarrow 4 \omega+4^{*} \bar{\omega} \quad 8_{c} \rightarrow 4 \bar{\omega}+4^{*} \omega \quad 8_{s} \rightarrow 6+\omega^{2}+\bar{\omega}^{2}
$$

with $\omega=e^{2 \pi i / k}$ playing the role of chemical potential or rather fugacity for the $\mathrm{SO}(2) \approx$ $\mathrm{U}(1)_{B}$ charge $Q$ commuting with $\mathrm{SO}(6)$ R-symmetry. One can introduce another three chemical potentials $\beta_{i}$ or fugacities $x_{i}=e^{i \beta_{i}}$ in order to keep track of the three Cartan's of $\mathrm{SO}(6) \approx \mathrm{SU}(4)$. We refrain from doing so here.

\subsection{Doubleton and higher spin gauge fields}

Doubleton representations can be obtained as tensor products of two singletons [46-48].

$$
\mathcal{D}(1 / 2,0) \otimes \mathcal{D}(1 / 2,0)=\oplus_{s=0}^{\infty} \mathcal{D}(\Delta=s+1, s)
$$

or

$$
\mathcal{D}(1,1 / 2) \otimes \mathcal{D}(1,1 / 2)=\mathcal{D}(\Delta=2, s=0)+\oplus_{s \neq 0}^{\infty} \mathcal{D}(\Delta=s+1, s)
$$

A consistent truncation, giving rise to minimal HS theories with even spins only, stems from restricting to symmetric tensors for bosons

$$
[\mathcal{D}(1 / 2,0) \otimes \mathcal{D}(1 / 2,0)]_{S}=\oplus_{k=0}^{\infty} \mathcal{D}(\Delta=2 k+1, s=2 k)
$$

or anti-symmetric for fermions

$$
[\mathcal{D}(1,1 / 2) \otimes \mathcal{D}(1,1 / 2)]_{A}=\mathcal{D}(\Delta=2, s=0)+\oplus_{k \neq 0}^{\infty} \mathcal{D}(\Delta=2 k+1, s=2 k)
$$


Odd spin states appear in the product with opposite symmetry

$$
[\mathcal{D}(1 / 2,0) \otimes \mathcal{D}(1 / 2,0)]_{A}=\oplus_{k=0}^{\infty} \mathcal{D}(\Delta=2 k+2, s=2 k+1)
$$

for bosons and

$$
[\mathcal{D}(1,1 / 2) \otimes \mathcal{D}(1,1 / 2)]_{S}=\oplus_{k=0}^{\infty} \mathcal{D}(\Delta=2 k+2, s=2 k+1)
$$

for fermions. Generators of the HS symmetry algebra can be realized as polynomials of bosonic oscillators $y_{\alpha}, y_{\dot{\alpha}}=\left(y_{\alpha}\right)^{\dagger}$ satisfying $\left[y_{\alpha}, y_{\beta}\right]=i \varepsilon_{\alpha \beta}$ and $\left[y_{\dot{\alpha}}, y_{\dot{\beta}}\right]=i \varepsilon_{\dot{\alpha} \dot{\beta}}$.

The supersymmetric extensions require the introduction of fermionic oscillators $\xi^{i}$ with $i=1, \ldots, \mathcal{N}$, satisfying $\left\{\xi^{i}, \xi^{j}\right\}=\delta^{i j}$. The resulting HS superalgebra denoted by $\operatorname{shs}^{E}(\mathcal{N} \mid 4)$ contains $O \operatorname{sp}(\mathcal{N} \mid 4)$ whose bosonic generators span $\mathrm{SO}(3,2) \cong \mathrm{Sp}(4, R)$ (conformal group) and $\mathrm{SO}(\mathcal{N})$ R-symmetry [38-40].

In particular for $\mathcal{N}=8$, with $\mathrm{SO}(8)$ R-symmetry, $\operatorname{Osp}(8 \mid 4)$ is the maximal finite dimensional subalgebra of the HS gauge algebra $s h s^{E}(8 \mid 4)$, which is a Lie superalgebra. The relevant super-singleton consists in ${ }^{3}$

$$
\widehat{\mathcal{D}}_{\mathcal{N}=8}=\mathcal{D}\left(1 / 2,0 ; \boldsymbol{8}_{v}\right) \oplus \mathcal{D}\left(1,1 / 2 ; \mathbf{8}_{c}\right)
$$

The (graded) symmetric product of two singletons $\left[\widehat{\mathcal{D}}_{\mathcal{N}=8} \otimes \widehat{\mathcal{D}}_{\mathcal{N}=8}\right]_{\hat{S}}$ yields

$$
\begin{aligned}
&\left\{\left[\mathcal{D}\left(1 / 2,0 ; \boldsymbol{8}_{v}\right) \oplus \mathcal{D}\left(1,1 / 2 ; \mathbf{8}_{c}\right)\right] \otimes\left[\mathcal{D}\left(1 / 2,0 ; \boldsymbol{8}_{v}\right) \oplus \mathcal{D}\left(1,1 / 2 ; \mathbf{8}_{c}\right)\right]\right\}_{\hat{S}}= \\
& \mathcal{D}\left(1,0 ; \mathbf{1}+\mathbf{3 5}_{v}\right) \oplus \mathcal{D}\left(2,0 ; \mathbf{1}+\mathbf{3 5}_{c}\right) \oplus_{k} \mathcal{D}\left(k+\frac{3}{2}, k+\frac{1}{2} ; \boldsymbol{8}_{s}+\mathbf{5 6}_{s}\right) \\
& \oplus_{k \neq 0} \mathcal{D}\left(2 k+1,2 k ; \mathbf{1}+\mathbf{3 5}_{v}+\mathbf{1}+\mathbf{3 5}_{c}\right) \oplus_{k} \mathcal{D}(2 k+2,2 k+1 ; \mathbf{2 8}+\mathbf{2 8})
\end{aligned}
$$

It is reassuring to recognize above the 'massless' states of $\mathcal{N}=8$ gauged supergravity on $A d S_{4}$. The remaining states with spin $s \leq 2$ belong to the 'short' Konishi multiplet and a 'semishort' multiplet with spin ranging from 2 to 6 [49-51]. Holography allows to relate AdS compactifications of supergravity and superstring theories to singleton field theories on the 3-d boundary. As a first step, these field theories can be constructed on the boundary of AdS as free superconformal theories. A remarkable property of singletons is that the symmetric product of two super-singletons gives an infinite tower of massless higher spin states. In the limit $\lambda \rightarrow 0$, all higher spin states become massless. After turning on interactions, a pantagruelic Higgs mechanism, named Grande Bouffe in [52-55], takes place. All but a handful of HS gauge fields become massive after 'eating' lowest spin states. The boundary counterpart of this phenomenon is the appearance of anomalous dimensions for HS currents and their superpartners. One should keep in mind that genuinely massive states are already present in the spectrum at $\lambda \rightarrow 0$ and arise in the product of three and more singletons.

\footnotetext{
${ }^{3}$ Different conventions for the $\mathrm{SO}(8)$ representations of bosons and fermions appear in the literature which are related to the present one, chosen for compatibility with our previous analysis, by $\mathrm{SO}(8)$ triality.
} 
Interacting theories for massless HS gauge fields, thus only describing the doubleton, have been proposed by Vasiliev [41, 44] that capture some aspects of the holographic correspondence in the extremely stringy (high AdS curvature) regime. Only vague glimpses of an interacting theory incorporating the Grande Bouffe have been offered so far [52-55].

Barring these subtle issues, let us discuss how to perform a $\mathbb{Z}_{k}$ projection of the spectrum giving rise to an $\mathcal{N}=6 \mathrm{HS}$ supergravity in $A d S_{4}$. In the limit $k \rightarrow \infty$ only $\mathrm{SO}(2)$ singlets survive

$$
\begin{aligned}
& \left\{\left[\mathcal{D}\left(1 / 2,0 ; \boldsymbol{8}_{v}\right) \oplus \mathcal{D}\left(1,1 / 2 ; \boldsymbol{8}_{c}\right)\right]\right\}_{\mathrm{SO}(2) \text { singlets }}^{\otimes 2_{\hat{s}}}= \\
& \quad \mathcal{D}(1,0 ; \mathbf{1}+\mathbf{1 5}) \oplus \mathcal{D}(2,0 ; \mathbf{1}+\mathbf{1 5}) \oplus_{k} \mathcal{D}\left(k+\frac{3}{2}, k+\frac{1}{2} ; \mathbf{6}+\mathbf{6}+\mathbf{1 0}+\mathbf{1 0}^{*}\right) \\
& \quad \oplus_{s \neq 0} \mathcal{D}(s+1, s ; \mathbf{1}+\mathbf{1 5}+\mathbf{1}+\mathbf{1 5})
\end{aligned}
$$

where indicated in bold-face are the surviving representations of the $\mathrm{SO}(6)$ R-symmetry. Candidate bosonic HS operators on the boundary in the $\mathbf{1}+\mathbf{1 5}$ of $\mathrm{SO}(6)$ are

$$
\mathcal{J}_{\mu_{1} \ldots \mu_{s}{ }_{j}}{ }=X^{i} \partial_{\mu_{1}} \partial_{\mu_{2}} \ldots \partial_{\mu_{s}} \bar{X}_{j}+\bar{\Psi}^{i} \gamma_{\mu_{1}} \partial_{\mu_{2}} \ldots \partial_{\mu_{s}} \Psi_{j}+\ldots
$$

where dots stand for symmetrization and subtraction of the traces and the coefficients of the linear combination are to be chosen appropriately.

At finite $k$ and $\lambda$, states with $\mathrm{SO}(2)$ charges $Q=k n$ survive. One can exploit orbifold technique to deduce the 'free' spectrum. ${ }^{4}$

The partition function or rather Witten index for the super-singleton of $O S p(8 \mid 4)$ reads:

$$
\mathcal{Z}_{\square}=\frac{8 q}{(1+q)^{2}}
$$

the $\mathbb{Z}_{k}$ projection reads

$$
\mathcal{Z}_{\square}^{\mathbb{Z}_{k}}=\frac{1}{k} \sum_{r=0}^{k-1} \mathcal{Z}_{\square}^{(r)}
$$

where

$$
\mathcal{Z}_{\square}^{(r)}=\frac{\left(4 \omega^{r}+4 \bar{\omega}^{r}\right) q}{(1+q)^{2}}
$$

with $\omega=e^{2 \pi i / k}$. Clearly $\mathcal{Z}_{\square}^{\mathbb{Z}_{k}}=0$ since $\Sigma_{r=0}^{k-1} \omega^{r}=0$.

A non-trivial spectrum arises from the doubleton partition function. Prior to the $\mathbb{Z}_{k}$ projection one has

$$
\mathcal{Z}_{\square}=\frac{1}{2}\left(\mathcal{Z}_{\square}^{2}(q)+\mathcal{Z}_{\square}\left(q^{2}\right)\right)=4 q^{2}\left(8(1+q)^{-4}+\left(1+q^{2}\right)^{-2}\right)
$$

for the (graded) symmetric doubleton, giving rise to precisely the spectrum of $h s(8 \mid 4)$ discussed above.

\footnotetext{
${ }^{4}$ Although $k$ is finite, one can take $k \gg N$, so that $\lambda \ll 1$, in order to identify states that eventually become massive.
} 


\begin{tabular}{|l|l|l|l|l|}
\hline$s \backslash h$ & 0 & 1 & 2 & 3 \\
\hline 0 & 70 & $1+1$ & & \\
\hline$\frac{1}{2}$ & 56 & 8 & & \\
\hline 1 & 28 & 28 & & \\
\hline$\frac{3}{2}$ & 8 & 56 & & \\
\hline 2 & 1 & 70 & 1 & \\
\hline$\frac{5}{2}$ & & 56 & 8 & \\
\hline 3 & & 28 & 28 & \\
\hline$\frac{7}{2}$ & & 8 & 56 & \\
\hline 4 & & 1 & 70 & 1 \\
\hline$\ldots$ & & & $\ldots$ & $\ldots$ \\
\hline
\end{tabular}

Table 3. $\mathcal{N}=8 h s(8 \mid 4) \supset O s p(8 \mid 4)$.

Performing the $\mathbb{Z}_{k}$ projection on the symmetric doubleton one finds

$$
\begin{aligned}
\mathcal{Z}_{\square}^{\mathbb{Z}_{k}} & =\frac{1}{2 k} \sum_{r}\left(\mathcal{Z}_{\square}^{(r)}(q, \omega)^{2}+\mathcal{Z}_{\square}^{(r)}\left(q^{2}, \omega^{2}\right)\right) \\
& =4 q^{2}\left[4\left(1+\sum_{r} \frac{\omega^{2 r}+\bar{\omega}^{2 r}}{2 k}\right)(1+q)^{-4}+\sum_{r} \frac{\omega^{2 r}+\bar{\omega}^{2 r}}{2 k}\left(1+q^{2}\right)^{-2}\right]
\end{aligned}
$$

for the (graded) symmetric doubleton, giving rise to precisely the 'massless' HS gauge fields of $h s(6 \mid 4)$ for $k \neq 2$ and $h s(8 \mid 4)$ for $k=1,2$, as expected $Z_{H S}=Z_{\square}$ ! Indeed

$$
\mathcal{Z}_{H S}=\frac{36\left(q^{2}+q^{4}\right)+72 \sum_{s=2 k \neq 0} F_{s}(q)+56 \sum_{s=2 k+1} F_{s}(q)-64 \sum_{s=k+\frac{1}{2}} F_{s}(q)}{\left(1-q^{2}\right)^{3}}
$$

with $F_{s}(q)=(2 s+1) q^{2(s+1)}-(2 s-1) q^{2(s+1)+2}$ taking into account the presence of null descendants for conserved spin $s$ currents of dimension $\Delta=s+1$. The relevant characters read

$$
\mathcal{X}_{s}^{\Delta=s+1}=\frac{q^{2 \Delta}(2 s+1)-q^{2(\Delta+1)}(2 s-1)}{\left(1-q^{2}\right)^{3}}=\frac{q^{2 \Delta}\left[\chi_{s}(\alpha)-q^{2} \chi_{s-1}(\alpha)\right]}{\left(1-q^{2}\right)\left(1-2 q^{2} \cos \alpha+q^{4}\right)}
$$

up to some $\mathrm{SO}(8)$ multiplicity $d_{(\ell, \ldots)}^{\mathrm{SO}(8)}$. Character formulae for $O s p(\mathcal{N} \mid 4)$ unitary irreducible representations can be found in [56].

The situation is summarized in the following tables, where $s$ denotes spin and $h$ the 'string' level. 


\begin{tabular}{|l|l|l|l|l|}
\hline$s \backslash h$ & 0 & 1 & 2 & 3 \\
\hline 0 & $15+15$ & $1+1$ & & \\
\hline$\frac{1}{2}$ & $10+10^{*}+6$ & 6 & & \\
\hline 1 & $15+1$ & $15+1$ & & \\
\hline$\frac{3}{2}$ & 6 & $10+10^{*}+6$ & & \\
\hline 2 & 1 & $15+15$ & 1 & \\
\hline$\frac{5}{2}$ & & $10+10^{*}+6$ & 6 & \\
\hline 3 & & $15+1$ & $15+1$ & \\
\hline$\frac{7}{2}$ & & 6 & $10+10^{*}+6$ & \\
\hline 4 & & 1 & $15+15$ & 1 \\
\hline$\frac{9}{2}$ & & & $10+10^{*}+6$ & 6 \\
\hline 5 & & & $15+1$ & $15+1$ \\
\hline$\frac{11}{2}$ & & & 6 & $10+10^{*}+6$ \\
\hline 6 & & & $1+1$ & $15+15$ \\
\hline$\ldots$ & & & $\ldots$ & $\ldots$ \\
\hline
\end{tabular}

Table 4. $\mathrm{SO}(2)$ neutral HS for $\mathcal{N}=6: h s(6 \mid 4) \supset O s p(6 \mid 4)$.

The decomposition into charged sectors reads

$$
\begin{aligned}
\mathcal{Z}_{\square}= & \frac{1}{\left(1-q^{2}\right)\left(1-2 q^{2} \cos \alpha+q^{4}\right)}\left\{\left[10\left(\omega^{2}+\omega_{c}^{2}\right)+16\right]\left(q^{2}+q^{4}\right) \chi_{0}(y)\right. \\
& +\sum_{j \in 1,3, \ldots}\left[12\left(\omega^{2}+\omega_{c}^{2}\right)+32\right]\left[\chi_{j}(y) q^{2(j+1)}-\chi_{j-1}(y) q^{2(j+1)+2}\right] \\
& +\sum_{j \in 2,4, \ldots}\left[20\left(\omega^{2}+\omega_{c}^{2}\right)+32\right]\left(\chi_{j}(y) q^{2(j+1)}-\chi_{j-1}(y) q^{2(j+1)+2}\right) \\
& \left.-\sum_{j \in 1 / 2,3 / 2, \ldots} 16\left(\omega+\omega_{c}\right)^{2}\left(\chi_{j}(y) q^{2(j+1)}-\chi_{j-1}(y) q^{2(j+1)+2}\right)\right\} .
\end{aligned}
$$

\subsection{Tripletons and higher $n$-pletons}

For higher multipletons one has to resort to Polya theory [52-55]. Consider a set of 'words' $A, B, \ldots$ of $n$ 'letters' chosen within the alphabet $\left\{a_{i}\right\}$ with $i=1, \ldots p$. When $p \rightarrow \infty$, let us denote by $\mathcal{Z}_{1}(q)$ the single letter 'partition function'. Let also $G$ be a group action defining the equivalence relation $A \sim B$ for $A=g B$ with $g$ an element of $G \subset S_{n}$. Elements $g \in S_{n}$ can be divided into conjugacy classes $[g]=(1)^{b_{1}} \ldots(n)^{b_{n}}$, according to the numbers $\left\{b_{k}(g)\right\}$ of cycles of length $k$. Polya theorem states that the set of inequivalent words are 


\begin{tabular}{|l|l|l|l|l|}
\hline$s \backslash h$ & 0 & 1 & 2 & \\
\hline 0 & $\left(10+10^{*}\right)_{ \pm 2}$ & & & \\
\hline$\frac{1}{2}$ & $15_{ \pm 2}$ & $1_{ \pm 2}$ & & \\
\hline 1 & $6_{ \pm 2}$ & $6_{ \pm 2}$ & & \\
\hline$\frac{3}{2}$ & $1_{ \pm 2}$ & $15_{ \pm 2}$ & & \\
\hline 2 & & $\left(10+10^{*}\right)_{ \pm 2}$ & & \\
\hline$\frac{5}{2}$ & & $15_{ \pm 2}$ & $1_{ \pm 2}$ & \\
\hline 3 & & $6_{ \pm 2}$ & $6_{ \pm 2}$ & \\
\hline$\frac{7}{2}$ & & $1_{ \pm 2}$ & $15_{ \pm 2}$ & \\
\hline 4 & & & $\left(10+10^{*}\right)_{ \pm 2}$ & \\
\hline$\ldots$ & & & $\ldots$ & $\ldots$ \\
\hline
\end{tabular}

Table 5. Charged HS for $\mathcal{N}=6: h s(8 \mid 4) / h s(6 \mid 4) \supset O s p(8 \mid 4) / O s p(6 \mid 4)$.

generated by the formula

$$
\mathcal{Z}_{n}^{G}=\frac{1}{|G|} \sum_{g \in G} \prod_{k=1}^{n} \mathcal{Z}_{1}\left(q^{k}\right)^{b_{k}(g)}
$$

In particular, for the cyclic group $G=Z_{n}$, conjugacy classes are $[g]=(d)^{n / d}$ for each divisor $d$ of $n$. The number of elements in a given conjugacy class labelled by $d$ is given by Eulers totient function $\mathcal{E}(d)$, equal to the number of integers relatively prime to $d$. For $d=1$ one defines $\mathcal{E}(1)=1$.

$$
\mathcal{Z}_{n}^{Z_{n}}=\frac{1}{n} \sum_{d \mid n} \mathcal{E}(d) \mathcal{Z}_{1}\left(q^{d}\right)^{n / d}
$$

For the full symmetric group one has

$$
\mathcal{Z}_{n}^{S_{n}}=\frac{1}{n !} \sum_{n_{r}: \sum_{r} r n_{r}=n} \frac{n !}{\prod_{r} r^{n_{r}} n_{r} !} \prod_{r} \mathcal{Z}_{1}\left(q^{r}\right)^{n_{r}}
$$

Let us consider the product of three singletons.

$$
\mathcal{Z}_{\square}^{3}=\mathcal{Z}_{\square \times \square \times \square} \rightarrow \mathcal{Z}_{\square \square}+\mathcal{Z}_{\square}+\mathcal{Z}_{\square}
$$

There are thus three kinds of tri-pletons.

The totally symmetric tripleton is coded in the partition function

$$
\mathcal{Z}_{\square \square}=\frac{1}{6}\left(\mathcal{Z}_{\square}^{3}(u)+3 \mathcal{Z}_{\square}(u) \mathcal{Z}_{\square}\left(u^{2}\right)+2 \mathcal{Z}_{\square}\left(u^{3}\right)\right)
$$

where $u$ collectively denotes the 'fugacities' $q, y=e^{i \alpha}, \omega \approx t, \ldots$. 
For the cyclic tripleton one has

$$
\mathcal{Z}_{\text {cycl }}=\mathcal{Z}_{\square \square}+\mathcal{Z}_{\boxminus}=\frac{1}{3}\left(\mathcal{Z}_{\square}^{3}(u)+2 \mathcal{Z}_{\square}\left(u^{3}\right)\right)
$$

For totally anti-symmetric tripletons one finds

$$
\mathcal{Z}_{\boxminus}=\mathcal{Z}_{c y c l}-\mathcal{Z}_{\square \square}=\frac{1}{6}\left(\mathcal{Z}_{\square}^{3}(u)+2 \mathcal{Z}_{\square}\left(u^{3}\right)-3 \mathcal{Z}_{\square}(u) \mathcal{Z}_{\square}\left(u^{2}\right)\right.
$$

while for mixed symmetry, incompatible with the cyclicity of the trace, one eventually finds

$$
\mathcal{Z}_{\square}=\mathcal{Z}_{\square}^{3}(u)-\frac{1}{3} \mathcal{Z}_{\square}^{3}(u)-\frac{2}{3} \mathcal{Z}_{\square}\left(u^{3}\right)=\frac{2}{3}\left(\mathcal{Z}_{\square}^{3}(u)-\mathcal{Z}_{\square}\left(u^{3}\right)\right)
$$

Recalling the singleton partition function

$$
\begin{aligned}
\mathcal{Z}_{\square}(q, \alpha, \omega) & =\frac{\left(4 \omega+4^{*} \bar{\omega}\right) q\left(1+q^{2}\right)}{\left(1-2 q^{2} \cos \alpha+q^{4}\right)}-\frac{\left(4 \bar{\omega}+4^{*} \omega\right) q^{2} \chi_{\frac{1}{2}}(\alpha)}{\left(1-2 q^{2} \cos \alpha+q^{4}\right)} \\
& =\frac{4(\omega+\bar{\omega}) q}{\left(1-2 q^{2} \cos \alpha+q^{4}\right)}\left[1+q^{2}-\chi_{\frac{1}{2}}(\alpha) q\right]
\end{aligned}
$$

where $\omega=e^{2 \pi i / k}$ and $\chi_{\frac{1}{2}}(\alpha)=t r_{1 / 2} \exp \left(i \alpha J_{3}\right)$, one eventually finds

$$
\begin{aligned}
\mathcal{Z}_{\square \square}=\frac{1}{6}\left(\frac{4^{3}(\omega+\bar{\omega})^{3} q^{3}\left(1+q^{2}-q \chi_{\frac{1}{2}}(\alpha)\right)^{3}}{\left(1-2 q^{2} \cos \alpha+q^{4}\right)^{3}}+\right. \\
\\
3 \frac{4(\omega+\bar{\omega}) q 4\left(\omega^{2}+\bar{\omega}^{2}\right) q^{2}\left(1+q^{2}-q \chi_{\frac{1}{2}}(\alpha)\right)\left(1+q^{4}-q^{2} \chi_{\frac{1}{2}}(2 \alpha)\right)}{\left(1-2 q^{2} \cos \alpha+q^{4}\right)\left(1-2 q^{4} \cos \alpha+q^{8}\right)}+ \\
\left.2 \frac{4\left(\omega^{3}+\bar{\omega}^{3}\right) q^{3}\left(1+q^{6}-q^{3} \chi_{\frac{1}{2}}(3 \alpha)\right)}{\left(1-2 q^{6} \cos \alpha+q^{12}\right)}\right)
\end{aligned}
$$

for the totally symmetric tripleton. Let us analyze the spectrum arising in this case. Except for the 1/2 BPS states, we will consider later on, only 'massive' representations above the unitary bound, whose characters read

$$
\mathcal{X}_{s}^{\Delta \neq s+1}=\frac{q^{2 \Delta} \chi_{s}(\alpha)}{\left(1-q^{2}\right)\left(1-2 q^{2} \cos \alpha+q^{4}\right)} \rightarrow_{\alpha \rightarrow 0} \frac{q^{2 \Delta}(2 s+1)}{\left(1-q^{2}\right)^{3}}
$$

appear in the decomposition

$$
\mathcal{Z}_{\square \square}(q, \alpha, \omega)=\sum_{s, \Delta, Q} c(s, \Delta, Q) \frac{q^{2 \Delta} \chi_{s}(\alpha) \omega^{Q}}{\left(1-q^{2}\right)\left(1-2 q^{2} \cos \alpha+q^{4}\right)}
$$

Indeed it is easy to see that no current like (twist $\tau=1$ ) fields appear beyond the doubleton, since the twist

$$
\tau=\Delta-s=\frac{n_{X}}{2}+n_{\partial}+n_{\Psi}-n_{\partial}-\frac{n_{\Psi}}{2}=\frac{n_{X}}{2}+\frac{n_{\Psi}}{2}>1
$$

whenever $n_{X}+n_{\Psi}>2$. 
Using orthogonality of the $\mathrm{SU}(2)$ characters

$$
\frac{1}{\pi} \int_{0}^{2 \pi} \chi_{s}(\alpha) \chi_{s^{\prime}}(\alpha) \sin ^{2} \frac{\alpha}{2} d \alpha=\delta_{2 s+1,2 s^{\prime}+1}
$$

one can decompose the partition function according to

$$
\sum_{Q, \Delta} \frac{c(s, \Delta, Q) \omega^{Q} q^{2 \Delta}}{\left(1-q^{2}\right)}=\frac{1}{\pi} \int_{0}^{2 \pi}\left(1-2 q^{2} \cos \alpha+q^{4}\right) \sin ^{2} \frac{\alpha}{2} \chi_{s}(\alpha) \mathcal{Z}_{\square \square}(q, \alpha, \omega) d \alpha
$$

It is clear that only states with charge $Q= \pm 3, \pm 1$ are present in the tri-pleton spectrum. Setting $y=e^{i \alpha}$, for states with $Q= \pm 1$ one finds

$$
\begin{aligned}
\mathcal{Z}_{\square}^{Q= \pm 1}= & \sum_{k=0}^{\infty}\left[(40+256 k) q^{4 k+3}+(104+256 k) q^{4 k+5}\right] \chi_{2 k}(y) \\
& -\left[(104+256 k) q^{4 k+4}+(152+256 k) q^{4 k+6}\right] \chi_{2 k+\frac{1}{2}}(y) \\
& +\left[(152+256 k) q^{4 k+5}+(216+256 k) q^{4 k+7}\right] \chi_{2 k+1}(y) \\
& -\left[(216+256 k) q^{4 k+6}+(296+256 k) q^{4 k+8}\right] \chi_{2 k+\frac{3}{2}}(y)
\end{aligned}
$$

these states are always projected out by $\mathbb{Z}_{k}$ since $\pm 1 \neq n k$. For states with $Q= \pm 3$ one finds instead

$$
\begin{aligned}
\mathcal{Z}_{\square \bigsqcup}^{Q= \pm 3}= & \sum_{k=0}^{\infty}\left[\left[(20+256 k) q^{12 k+3}+(40+256 k) q^{12 k+5}\right] \chi_{6 k}(y)\right. \\
& -\left[(40+256 k) q^{12 k+4}+(44+256 k) q^{12 k+6}\right] \chi_{6 k+\frac{1}{2}}(y) \\
& +\left[(44+256 k) q^{12 k+5}+(68+256 k) q^{12 k+7}\right] \chi_{6 k+1}(y) \\
& -\left[(68+256 k) q^{12 k+6}+(104+256 k) q^{12 k+8}\right] \chi_{6 k+\frac{3}{2}}(y) \\
& +\left[(104+256 k) q^{12 k+7}+(124+256 k) q^{12 k+9}\right] \chi_{6 k+2}(y) \\
& -\left[(124+256 k) q^{12 k+8}+(132+256 k) q^{12 k+10}\right] \chi_{6 k+\frac{5}{2}}(y) \\
& +\left[(132+256 k) q^{12 k+9}+(152+256 k) q^{12 k+11}\right] \chi_{6 k+3}(y) \\
& -\left[(152+256 k) q^{12 k+10}+(188+256 k) q^{12 k+12}\right] \chi_{6 k+\frac{7}{2}}(y) \\
& +\left[(188+256 k) q^{12 k+11}+(212+256 k) q^{12 k+13}\right] \chi_{6 k+4}(y) \\
& -\left[(212+256 k) q^{12 k+12}+(216+256 k) q^{12 k+14}\right] \chi_{6 k+\frac{9}{2}}(y) \\
& +\left[(216+256 k) q^{12 k+13}+(236+256 k) q^{12 k+15}\right] \chi_{6 k+5}(y) \\
& \left.-\left[(236+256 k) q^{12 k+14}+(276+256 k) q^{12 k+16}\right] \chi_{6 k+\frac{11}{2}}(y)\right]
\end{aligned}
$$

These states survive only for $k=3$, i.e. $\mathbb{Z}_{3}$ projection. It is amusing to observe how the number of representations of given spin $s=6 k+\frac{n}{2}$ grows with $k$ at the rate $256 k$ for any $n$. 
This is due to the possible distributions of derivatives among three fields up to symmetries and total derivatives and to the structure of higher spin supermultiplets $[56,57]$.

For higher multi-pletons the analysis is similar. It is clear that only states with charge $Q= \pm n, \pm(n-2), \ldots$ are present in the n-pleton spectrum. In particular $Q=0$ states are only present when $n$ is even as already observed. We defer a detailed analysis to the future. For the time being let us only display the partition functions for the cyclic tetrapleton

$$
\mathcal{Z}_{4, c y c l}=\frac{1}{4}\left(\mathcal{Z}_{\square}(q)^{4}+\mathcal{Z}_{\square}^{2}\left(q^{2}\right)+2 \mathcal{Z}_{\square}\left(q^{4}\right)\right)
$$

and for the totally symmetric tetrapleton

$$
\mathcal{Z}_{\square \square \square}=\frac{1}{4 !}\left(\mathcal{Z}_{\square}^{4}(q)+6 \mathcal{Z}_{\square}^{2}(q) \mathcal{Z}_{\square}\left(q^{2}\right)+3 \mathcal{Z}_{\square}^{2}\left(q^{2}\right)+8 \mathcal{Z}_{\square}\left(q^{3}\right) \mathcal{Z}_{\square}(q)+6 \mathcal{Z}_{\square}\left(q^{4}\right)\right)
$$

The $\mathbb{Z}_{k}$ projection on n-pletons reads

$$
\mathcal{Z}_{n}^{\mathbb{Z}_{k}}=\frac{1}{k} \sum_{r} \mathcal{Z}_{n}^{(r)}\left(q, \omega^{r}\right)
$$

and corresponds to keeping only states with $Q=k n$ i.e. integer multiples of $k$.

\subsection{KK excitations}

Let us now focus on the KK excitations, which deserve a separate treatment. One can indeed write down the single-particle partition function on $S^{7}$, decompose it into supercharacters and identify the $\mathrm{SO}(2)$ charge sectors, relevant for the subsequent $\mathbb{Z}_{k}$ projection i.e. compactification on $\mathbb{C P}^{3}$.

Introducing a chemical potential for the charge $Q\left(t^{Q}\right)$, the super-character of an ultrashort 1/2 BPS representation of $O s p(8 \mid 4)$ reads:

$$
\begin{aligned}
\mathcal{X}_{\ell}^{1 / 2 B P S}(q, t)= & \frac{t^{-2-\ell} q^{2+\ell}}{6\left(1-t^{2}\right)^{5}(1+q)^{3}}\left[\ell^{3}\left(-1+t^{2}\right)^{2}(-1+q)^{3}\right. \\
& \times\left(t^{6+2 \ell}\left(t^{2}-q\right)^{2}-\left(-1+t^{2} q\right)^{2}\right)-6 \ell^{2}\left(-1+t^{2}\right)(-1+q)^{2} \\
& \times\left(t^{6+2 \ell}\left(t^{2}-q\right)^{2}\left(-3+2 t^{2}+q\right)+\left(2+t^{2}(-3+q)\right)\left(-1+t^{2} q\right)^{2}\right) \\
& +6 t^{6+2 \ell}\left(t^{2}-q\right)^{2}\left(-35+q(35+(-9+q) q)+2 t^{4}\left(-5+q^{2}\right)\right. \\
& \left.+t^{2}(35+q(-13+(-7+q) q))\right)-\left(2\left(-5+q^{2}\right)\right. \\
& \left.+t^{4}(-35+q(35+(-9+q) q))+t^{2}(35+q(-13+(-7+q) q))\right) \\
& \times 6\left(-1+t^{2} q\right)^{2}-\ell(-1+q)\left(t^{6+2 \ell}\left(t^{2}-q\right)^{2}(-107+(70-11 q) q\right. \\
& \left.+t^{4}(-47+(-2+q) q)-2 t^{2}(-71+q(22+q))\right)+\left(-1+t^{2} q\right)^{2} \\
& \left.\left.\times\left(47-(-2+q) q+2 t^{2}(-71+q(22+q))+t^{4}(107+q(-70+11 q))\right)\right)\right]
\end{aligned}
$$


For $\ell=0$, corresponding to the gauged supergravity multiplet, there is further shortening (null descendants) due to the presence of conserved 'currents' i.e. stress-tensor, $\mathrm{SO}(8)$ vector currents and $\boldsymbol{8}_{s}$ supercurrents. Taking this into account one finds the following super-character

$$
\begin{aligned}
\mathcal{X}_{\ell=0}^{1 / 2 B P S}(q)=\frac{1}{\left(1-q^{2}\right)^{3}}[ & \left(10 t^{2}+15+10 t^{-2}\right) q^{2}- \\
& 2\left(15 t^{2}+10+6+10+15 t^{-2}\right) q^{3}+ \\
& \left(10 t^{2}+15+10 t^{-2}+3\left(6 t^{2}+15+1+6 t^{-2}\right)\right) q^{4}- \\
& 4\left(t^{2}+6+t^{-2}\right) q^{5}-\left(6 t^{2}+15+1-5+6 t^{-2}\right) q^{6}+ \\
& \left.2\left(t^{2}+6+t^{-2}\right) q^{7}-3 q^{8}\right]
\end{aligned}
$$

the denominator takes into account derivatives (descendants). Quite remarkably this formula coincides with the previous one when $\ell=0$.

After some algebra, putting $t=1$, one finds

$$
\mathcal{X}_{\ell=0}^{1 / 2 B P S}(q)=\frac{q^{2}\left(3 q^{3}-7 q^{2}-7 q+35\right)}{(1+q)^{3}}
$$

a factor $(1-q)^{2}$ cancels between numerator and denominator meaning that not only $n_{b}=n_{f}$ and the sum with $\Delta^{1}$ vanishes but also the sum with $\Delta^{2}$ should vanish. This should be related to the absence of quantum corrections to the negative vacuum energy, i.e. cosmological constant in the bulk.

The $1 / 2$ BPS partition function is given by

$$
\mathcal{Z}_{1 / 2 B P S}^{\mathcal{N}=8}=\sum_{\ell} \mathcal{X}_{\ell}^{1 / 2 B P S}=\frac{35 q^{2}}{\left(1-q^{2}\right)^{2}}
$$

The simplicity of the result is due to 'miraculous' cancellations between bosonic and fermionic operators with the same scaling dimensions in different KK multiplets i.e. with different $\ell$ 's. This does not happen in $A d S_{5} / C F T_{4}$ holography, whereby (protected) bosonic operator have integer dimensions and (protected) fermionic operators have half-integer dimensions [42, 43, 52-57].

In order to perform the $\mathbb{Z}_{k}$ projection it is useful to decompose into $\mathrm{SO}(2)$ charge sectors according to

$$
\mathcal{Z}_{1 / 2 B P S}^{\mathcal{N}=8 \rightarrow \mathcal{N}=6}(q, t)=\frac{q^{2}\left[\left(1+q^{6}\right) P_{2}(t)-\left(q+q^{5}\right) P_{3}(t)+\left(q^{2}+q^{4}\right) P_{4}(t)-q^{3} P_{5}(t)\right]}{(1-q t)^{4}\left(1-q t^{-1}\right)^{4}(1+q)^{2}}
$$

where

$$
\begin{aligned}
& P_{2}(t)=10 t^{+2}+15+10 t^{-2} \\
& P_{3}(t)=20 t^{+3}+10 t^{+2}+64 t^{+1}+22+64 t^{-1}+10 t^{-2}+20 t^{-3}
\end{aligned}
$$




$$
\begin{aligned}
P_{4}(t)= & 15 t^{+4}+8 t^{+3}+104 t^{+2}+48 t^{+1}+175+48 t^{-1}+104 t^{-2}+8 t^{-3}+15 t^{-4} \\
P_{5}(t)= & 4 t^{+5}+2 t^{+4}+64 t^{+3}+40 t^{+2}+196 t^{+1}+88+ \\
& +196 t^{-1}+40 t^{-2}+64 t^{-3}+2 t^{-4}+4 t^{-5}
\end{aligned}
$$

Depending on the choice of $k$ one can recognize the surviving $1 / 2$ BPS states as those with $Q=k n$. In formulae one has to replace $t$ with $\omega^{r}$ and sum over $r=0, \ldots, k-1$.

\section{Conclusions}

We have re-analyzed the KK spectrum of $d=11$ supergravity on $S^{7}$ and $S^{7} / \mathbb{Z}_{k}$. The latter includes monopole operators dual to charged states in Type IIA on $\mathbb{C P}^{3}$. To this end we have presented some group theoretic methods for the decomposition of the $\mathrm{SO}(8)$ into $\mathrm{SO}(6) \times \mathrm{SO}(2)$ valid also for other cosets [58-60] where resolution of the mixing among various fluctuations should be possible on the basis of symmetry arguments. In particular, massless vectors associated to Killing vectors in generic flux vacua with isometries have been recently discussed in [61].

We have then considered higher spin symmetry enhancement. We have displayed the partition functions for singletons, doubletons and tripletons and discussed in details higher spin fields and 1/2 BPS states corresponding to KK excitations of $\mathcal{N}=6$ gauged supergravity. It would be worth pursuing the analysis to higher n-pletons and to cases with lower supersymmetry, yet based on internal coset manifolds.

\section{Acknowledgments}

Discussions with S. Ferrara, D. Forcella, F. Fucito, L. Lopez, J. F. Morales, R. Richter, A. V. Santini, D. Sorokin and L. Wulff are kindly acknowledged. M. B. and M. S. would like to particularly thank M. Naghdi for sharing his insights on the ABJM model and collaborating on related issues at an early stage of this project. This work was partially supported by the ERC Advanced Grant n.226455 "Superfields" and by the Italian MIUR-PRIN contract 2007-5ATT78 "Symmetries of the Universe and of the Fundamental Interactions". The work of R.P. was partially supported by the European Commission FP7 Programme Marie Curie Grant Agreement PIIF-GA-2008-221571, and the Institutional Partnership grant of the Humboldt Foundation of Germany.

\section{A Dimension formulae for $\mathrm{SO}(8)$}

General formula

$$
\begin{aligned}
d_{\left(\ell_{1}, \ell_{2}, \ell_{3}, \ell_{4}\right)}^{\mathrm{SO}(8)}= & \frac{1}{4320} \times\left(1+\ell_{1}\right)\left(1+\ell_{2}\right)\left(1+\ell_{3}\right)\left(1+\ell_{4}\right) \\
& \left(2+\ell_{1}+\ell_{2}\right)\left(2+\ell_{2}+\ell_{3}\right)\left(2+\ell_{2}+\ell_{4}\right)
\end{aligned}
$$




$$
\begin{aligned}
& \left(3+\ell_{1}+\ell_{2}+\ell_{3}\right)\left(3+\ell_{1}+\ell_{2}+\ell_{4}\right)\left(3+\ell_{2}+\ell_{3}+\ell_{4}\right) \\
& \left(4+\ell_{1}+\ell_{2}+\ell_{3}+\ell_{4}\right)\left(5+\ell_{1}+2 \ell_{2}+\ell_{3}+\ell_{4}\right)
\end{aligned}
$$

Specific cases (KK harmonics)

$$
\begin{aligned}
& d_{(\ell, 0,0,0)}^{\mathrm{SO}(8)}=\frac{1}{360}(1+\ell)(2+\ell)(3+\ell)^{2}(4+\ell)(5+\ell) \quad \leftrightarrow \quad Y_{N_{1}} \\
& d_{(\ell, 1,0,0)}^{\mathrm{SO}(8)}=\frac{1}{60}(1+\ell)(3+\ell)(4+\ell)^{2}(5+\ell)(7+\ell) \quad \leftrightarrow \quad Y_{N_{7}} \\
& d_{(\ell, 0,1,1)}^{\mathrm{SO}(8)}=\frac{1}{24}(1+\ell)(2+\ell)(4+\ell)^{2}(6+\ell)(7+\ell) \quad \leftrightarrow \quad Y_{N_{21}} \\
& d_{(\ell, 2,0,0)}^{\mathrm{SO}(8)}=\frac{1}{18}(1+\ell)(4+\ell)(5+\ell)^{2}(6+\ell)(9+\ell) \quad \leftrightarrow \quad Y_{N_{27}} \\
& d_{(\ell, 0,2,0)}^{\mathrm{SO}(8)}=d_{(\ell, 0,0,2)}^{\mathrm{SO}(8)}=\frac{1}{36}(1+\ell)(2+\ell)(3+\ell)(5+\ell)(6+\ell)(7+\ell) \leftrightarrow \quad Y_{N_{35}} \\
& d_{(\ell, 0,1,0)}^{\mathrm{SO}(8)}=d_{(\ell, 0,0,1)}^{\mathrm{SO}(8)}=\frac{1}{90}(1+\ell)(2+\ell)(3+\ell)(4+\ell)(5+\ell)(6+\ell) \\
& d_{(\ell, 1,1,0)}^{\mathrm{SO}(8)}=d_{(\ell, 1,0,1)}^{\mathrm{SO}(8)}=\frac{1}{18}(1+\ell)(3+\ell)(4+\ell)(5+\ell)(6+\ell)(8+\ell)
\end{aligned}
$$

\section{B Zero charge states}

In this appendix we list states with $Q=0$ in the KK towers of $S^{7}$ after the decomposition of $\mathrm{SO}(8)$ into $\mathrm{SO}(6) \times \mathrm{SO}(2)$.

Bosons:

$$
\begin{aligned}
(\ell, 0,0,0)_{\ell \geq 0} \rightarrow & {\left[0, \frac{\ell}{2}, \frac{\ell}{2}\right] } \\
(\ell, 1,0,0)_{\ell \geq 0} \rightarrow & {\left[0, \frac{\ell}{2}, \frac{\ell}{2}\right]+\left[0, \frac{\ell}{2}+1, \frac{\ell}{2}+1\right] } \\
& +\left[1, \frac{\ell}{2}+1, \frac{\ell}{2}-1\right]+\left[1, \frac{\ell}{2}-1, \frac{\ell}{2}+1\right] \\
(\ell-1,0,1,1)_{\ell \geq 1} \rightarrow & {\left[0, \frac{\ell}{2}+2, \frac{\ell}{2}-2\right]+\left[0, \frac{\ell}{2}-2, \frac{\ell}{2}+2\right]+\left[1, \frac{\ell}{2}+1, \frac{\ell}{2}-1\right] } \\
& +\left[1, \frac{\ell}{2}-1, \frac{\ell}{2}+1\right]+\left[0, \frac{\ell}{2}, \frac{\ell}{2}\right]+\left[0, \frac{\ell}{2}, \frac{\ell}{2}\right] \\
& +\left[1, \frac{\ell}{2}, \frac{\ell}{2}-2\right]+\left[1, \frac{\ell}{2}-2, \frac{\ell}{2}\right]+\left[2, \frac{\ell}{2}-1, \frac{\ell}{2}-1\right] \\
(\ell-2,2,0,0)_{\ell \geq 2} \rightarrow & {\left[2, \frac{\ell}{2}-3, \frac{\ell}{2}+1\right]+\left[1, \frac{\ell}{2}-2, \frac{\ell}{2}\right]+\left[1, \frac{\ell}{2}, \frac{\ell}{2}-2\right] } \\
& +\left[0, \frac{\ell}{2}, \frac{\ell}{2}\right]+\left[0, \frac{\ell}{2}-1, \frac{\ell}{2}-1\right]+\left[1, \frac{\ell}{2}-1, \frac{\ell}{2}+1\right] \\
& +\left[1, \frac{\ell}{2}+1, \frac{\ell}{2}-1\right]+\left[2, \frac{\ell}{2}-1, \frac{\ell}{2}-1\right]+\left[2, \frac{\ell}{2}+1, \frac{\ell}{2}-3\right]+\left[0, \frac{\ell}{2}+1, \frac{\ell}{2}+1\right]
\end{aligned}
$$




$$
\begin{aligned}
(\ell, 0,2,0)_{\ell \geq 0} \rightarrow & {\left[0, \frac{\ell}{2}-1, \frac{\ell}{2}+3\right]+\left[0, \frac{\ell}{2}+1, \frac{\ell}{2}+1\right]+\left[0, \frac{\ell}{2}+3, \frac{\ell}{2}-1\right] } \\
& +\left[1, \frac{\ell}{2}-1, \frac{\ell}{2}+1\right]+\left[1, \frac{\ell}{2}+1, \frac{\ell}{2}-1\right]+\left[2, \frac{\ell}{2}-1, \frac{\ell}{2}-1\right] \\
(\ell-2,0,0,2)_{\ell \geq 2} \rightarrow & {\left[0, \frac{\ell}{2}-1, \frac{\ell}{2}-1\right]+\left[0, \frac{\ell}{2}-3, \frac{\ell}{2}+1\right]+\left[1, \frac{\ell}{2}, \frac{\ell}{2}-2\right] } \\
& +\left[1, \frac{\ell}{2}-2, \frac{\ell}{2}\right]+\left[2, \frac{\ell}{2}-1, \frac{\ell}{2}-1\right]+\left[0, \frac{\ell}{2}+1, \frac{\ell}{2}-3\right]
\end{aligned}
$$

Fermions:

$$
\begin{aligned}
(\ell, 0,0,1)_{\ell \geq 0} \rightarrow & {\left[0, \frac{\ell}{2}-1, \frac{\ell}{2}+1\right]+\left[0, \frac{\ell}{2}+1, \frac{\ell}{2}-1\right]+\left[1, \frac{\ell}{2}, \frac{\ell}{2}\right] } \\
(\ell-1,0,1,0)_{\ell \geq 1} \rightarrow & {\left[0, \frac{\ell}{2}-1, \frac{\ell}{2}+1\right]+\left[0, \frac{\ell}{2}+1, \frac{\ell}{2}-1\right]+\left[1, \frac{\ell}{2}-1, \frac{\ell}{2}-1\right] } \\
(\ell-1,1,1,0)_{\ell \geq 1} \rightarrow & {\left[1, \frac{\ell}{2}-2, \frac{\ell}{2}+2\right]+2\left[1, \frac{\ell}{2}, \frac{\ell}{2}\right]+\left[1, \frac{\ell}{2}+2, \frac{\ell}{2}-2\right] } \\
& +\left[0, \frac{\ell}{2}, \frac{\ell}{2}+2\right]+\left[0, \frac{\ell}{2}+2, \frac{\ell}{2}\right]+\left[0, \frac{\ell}{2}-1, \frac{\ell}{2}+1\right] \\
& +\left[0, \frac{\ell}{2}+1, \frac{\ell}{2}-1\right]+\left[2, \frac{\ell}{2}-2, \frac{\ell}{2}\right]+\left[2, \frac{\ell}{2}, \frac{\ell}{2}-2\right]+\left[1, \frac{\ell}{2}-1, \frac{\ell}{2}-1\right] \\
(\ell-2,1,0,1)_{\ell \geq 2} \rightarrow & {\left[1, \frac{\ell}{2}-3, \frac{\ell}{2}+1\right]+2\left[1, \frac{\ell}{2}-1, \frac{\ell}{2}-1\right]+\left[1, \frac{\ell}{2}+1, \frac{\ell}{2}-3\right] } \\
& +\left[0, \frac{\ell}{2}-2, \frac{\ell}{2}\right]+\left[0, \frac{\ell}{2}, \frac{\ell}{2}-2\right]+\left[0, \frac{\ell}{2}-1, \frac{\ell}{2}+1\right] \\
& +\left[0, \frac{\ell}{2}+1, \frac{\ell}{2}-1\right]+\left[2, \frac{\ell}{2}-2, \frac{\ell}{2}\right]+\left[2, \frac{\ell}{2}, \frac{\ell}{2}-2\right]+\left[1, \frac{\ell}{2}, \frac{\ell}{2}\right]
\end{aligned}
$$

\section{Generating functions for $\mathrm{SO}(8)$ representations}

The generating function for multiplicities of the scalar spherical harmonics on $S^{7}$ is given by

$$
\mathcal{F}_{N_{1}}(q)=\frac{1+q}{(1-q)^{7}}
$$

The coefficient of $q^{\ell}$ gives the dimension of the $\mathrm{SO}(8)$ representation with Dynkin label $(\ell, 0,0,0)$.

The generating function for vector spherical harmonics with $\mathrm{SO}(8)$ Dynkin label $(\ell-$ $1,1,0,0)$ reads:

$$
\mathcal{F}_{N_{7}}(q)=\frac{\left(28-36 q+35 q^{2}-21 q^{3}+7 q^{4}-q^{5}\right) q}{(1-q)^{7}}
$$

For two-form spherical harmonics with $\mathrm{SO}(8)$ Dynkin label $(\ell-1,0,1,1)$ the generating function is:

$$
\mathcal{F}_{N_{21}}(q)=\frac{\left(56-42 q+22 q^{2}-7 q^{3}+q^{4}\right) q^{2}}{(1-q)^{7}}
$$


For second rank symmetric traceless harmonics the $\mathrm{SO}(8)$ Dynkin index is $(\ell, 2,0,0)$ and the generating function is given by the following formula:

$$
\mathcal{F}_{N_{27}}(q)=\frac{4\left(75-175 q+203 q^{2}-133 q^{3}+47 q^{4}-7 q^{5}\right) q^{2}}{(1-q)^{7}}
$$

Finally, for three-form spherical harmonics with $\mathrm{SO}(8)$ Dynkin label $(\ell-1,0,2,0)$ (or $(\ell-1,0,0,2))$ one has

$$
\mathcal{F}_{N_{35}}(q)=\frac{\left(35-21 q+7 q^{2}-q^{3}\right) q^{2}}{(1-q)^{7}}
$$

Let us complete the description with the spectrum of spinor spherical harmonics.

For gravitini with Dynkin labels $(\ell, 0,0,1)_{\ell \geq 0}$ and $(\ell-1,0,1,0)_{\ell \geq 1}$, the generating function is:

$$
\mathcal{F}_{\text {gravitini }}(q)=\frac{8 q}{(1-q)^{7}}
$$

For spinors with Dynkin labels $(\ell-1,1,1,0)_{\ell \geq 1}$ and $(\ell-2,1,0,1)_{\ell \geq 2}$ one has

$$
\mathcal{F}_{\text {spinor }}(q)=\frac{8 q^{2}\left(20-35 q+35 q^{2}-21 q^{3}+7 q^{4}-q^{5}\right)}{(1-q)^{7}} .
$$

\section{Generating functions for $\mathrm{SO}(6)$ representations}

In this appendix we present the decomposition of the $\mathrm{SO}(8)$ generating functions under $\mathrm{SO}(6) \times \mathrm{SO}(2)$. Below a factor of $\left(1-q t^{-1}\right)^{-4}(1-q t)^{-4}$ is always understood.

For $(\ell, 0,0,0)$ one has:

$$
\hat{\mathcal{F}}_{\text {graviton }}(q)=1-q^{2}
$$

For $(\ell, 1,0,0)$ one has:

$$
\begin{aligned}
\hat{\mathcal{F}}_{g b 1}(q, t)= & 6 t^{2}-4 t q-4 t^{3} q+q^{2}+t^{4} q^{2} \\
\hat{\mathcal{F}}_{g b 2}(q, t)= & 1-q^{2} \\
\hat{\mathcal{F}}_{g b 3}(q, t)= & 15+36 q^{2}-4 q^{3} t^{-3}-4 t^{3} q^{3}+16 q^{4}+q^{6}+\left(16 q^{2}+6 q^{4}\right) t^{-2}+ \\
& t^{2}\left(16 q^{2}+6 q^{4}\right)-\left(24 q+24 q^{3}+4 q^{5}\right) t^{-1}-t\left(24 q+24 q^{3}+4 q^{5}\right) \\
\hat{\mathcal{F}}_{g b 4}(q, t)= & 6 t^{-2}-4 q t^{-3}-4 q t^{-1}+q^{2}+q^{2} t^{-4}
\end{aligned}
$$


For $(\ell-1,0,1,1)$ one has:

$$
\begin{aligned}
\hat{\mathcal{F}}_{g b}^{1}(q, t)= & 4 t^{3} q-6 t^{2} q^{2}-t^{4} q^{2}+4 t q^{3}-q^{4} \\
\hat{\mathcal{F}}_{g b}^{2}(q, t)= & 4 t q-q^{2}-6 t^{2} q^{2}+4 t^{3} q^{3}-t^{4} q^{4} \\
\hat{\mathcal{F}}_{g b}^{3}(q, t)= & -35 q^{2}+4 t^{3} q^{3}-16 q^{4}-6 q^{4} t^{-2}-q^{6}-t^{2}\left(16 q^{2}+6 q^{4}\right)+ \\
& \left(24 q^{3}+4 q^{5}\right) t^{-1}+t\left(20 q+24 q^{3}+4 q^{5}\right) \\
\hat{\mathcal{F}}_{g b}^{4}(q, t)= & -35 q^{2}+4 q^{3} t^{-3}-16 q^{4}-6 t^{2} q^{4}-q^{6}-\left(16 q^{2}+6 q^{4}\right) t^{-2}+ \\
& t\left(24 q^{3}+4 q^{5}\right)+\left(20 q+24 q^{3}+4 q^{5}\right) t^{-1} \\
\hat{\mathcal{F}}_{g b}^{5}(q, t)= & 4 q t^{-1}-q^{2}-6 q^{2} t^{-2}+4 q^{3} t^{-3}-q^{4} t^{-4} \\
\hat{\mathcal{F}}_{g b}^{6}(q, t)= & 4 q t^{-3}-q^{2} t^{-4}-6 q^{2} t^{-2}+4 q^{3} t^{-1}-q^{4} \\
\hat{\mathcal{F}}_{g b}^{7}(q, t)= & 6 t^{2} q^{2}-4 t q^{3}-4 t^{3} q^{3}+q^{4}+t^{4} q^{4} \\
\hat{\mathcal{F}}_{g b}^{8}(q, t)= & 6 q^{4} t^{-2}+6 t^{2} q^{4}-q^{2}\left(20 q+4 q^{3}\right) t^{-1}-t q^{2}\left(20 q+4 q^{3}\right)+ \\
& q^{2}\left(20+15 q^{2}+q^{4}\right) \\
\hat{\mathcal{F}}_{g b}^{9}(q, t)= & 6 q^{2} t^{-2}-4 q^{3} t^{-3}-4 q^{3} t^{-1}+q^{4}+q^{4} t^{-4}
\end{aligned}
$$

For $(\ell-2,2,0,0)$ one has:

$$
\begin{aligned}
\hat{\mathcal{F}}_{s c 1}^{1}(q, t)= & 6 t^{2} q^{4}+6 t^{6} q^{4}-t^{3} q^{2}\left(20 q+4 q^{3}\right)-t^{5} q^{2}\left(20 q+4 q^{3}\right)+ \\
& t^{4} q^{2}\left(20+15 q^{2}+q^{4}\right) \\
\hat{\mathcal{F}}_{s c 1}^{2}(q, t)= & 6 t^{2} q^{2}-4 t q^{3}-4 t^{3} q^{3}+q^{4}+t^{4} q^{4} \\
\hat{\mathcal{F}}_{s c 1}^{3}(q, t)= & 6 q^{2} t^{-2}-4 q^{3} t^{-3}-4 q^{3} t^{-1}+q^{4}+q^{4} t^{-4} \\
\hat{\mathcal{F}}_{s c 1}^{4}(q, t)= & -1+4 q t^{-1}+4 t q-q^{2}-6 q^{2} t^{-2}-6 t^{2} q^{2}+4 q^{3} t^{-3}+4 t^{3} q^{3}- \\
& q^{4} t^{-4}-t^{4} q^{4} \\
\hat{\mathcal{F}}_{s c 1}^{5}(q, t)= & q^{2}-q^{4} \\
\hat{\mathcal{F}}_{s c 1}^{6}(q, t)= & -36 q^{2}-16 q^{4}-6 q^{4} t^{-2}-6 t^{6} q^{4}-q^{6}-t^{2}\left(6+32 q^{2}+12 q^{4}\right)+ \\
& t\left(24 q+28 q^{3}+4 q^{5}\right)+t^{3}\left(24 q+28 q^{3}+4 q^{5}\right)+\left(24 q^{3}+4 q^{5}\right) t^{-1}+ \\
& t^{5}\left(24 q^{3}+4 q^{5}\right)-t^{4}\left(36 q^{2}+16 q^{4}+q^{6}\right) \\
\hat{\mathcal{F}}_{s c 1}^{7}(q, t)= & -36 q^{2}-16 q^{4}-6 q^{4} t^{-6}-6 t^{2} q^{4}-q^{6}-\left(6+32 q^{2}+12 q^{4}\right) t^{-2}+ \\
& \left(24 q^{3}+4 q^{5}\right) t^{-5}+t\left(24 q^{3}+4 q^{5}\right)+\left(24 q+28 q^{3}+4 q^{5}\right) t^{-3}+ \\
& \left(24 q+28 q^{3}+4 q^{5}\right) t^{-1}-\left(36 q^{2}+16 q^{4}+q^{6}\right) t^{-4} \\
\hat{\mathcal{F}}_{s c 1}^{8}(q, t)= & 6 q^{4} t^{-2}+6 t^{2} q^{4}-q^{2}\left(20 q+4 q^{3}\right) t^{-1}-t q^{2}\left(20 q+4 q^{3}\right)+ \\
& q^{2}\left(20+15 q^{2}+q^{4}\right) \\
\hat{\mathcal{F}}_{s c 1}^{9}(q, t)= & 6 q^{4} t^{-6}+6 q^{4} t^{-2}-q^{2}\left(20 q+4 q^{3}\right) t^{-5}-q^{2}\left(20 q+4 q^{3}\right) t^{-3}+ \\
& q^{2}\left(20+15 q^{2}+q^{4}\right) t^{-4} \\
\hat{\mathcal{F}}_{s c 1}^{10}(q, t)= & -15-156 q^{2}-176 q^{4}-15 q^{4} t^{-4}-15 t^{4} q^{4}-31 q^{6}+ \\
& \left(24 q^{5}\right) t^{-3}+t^{3}\left(60 q^{3}+24 q^{5}\right)-\left(90 q^{2}+106 q^{4}+16 q^{6}\right) t^{-2}-
\end{aligned}
$$




$$
\begin{aligned}
& t^{2}\left(90 q^{2}+106 q^{4}+16 q^{6}\right)+\left(60 q+184 q^{3}+84 q^{5}+4 q^{7}\right) t^{-1}+ \\
& t\left(60 q+184 q^{3}+84 q^{5}+4 q^{7}\right)
\end{aligned}
$$

For $(\ell, 0,2,0)$ one has:

$$
\begin{aligned}
\hat{\mathcal{F}}_{s c 2}^{1}(q, t)= & -4 t^{3} q+15 q^{2}-4 q^{3} t^{-1}+q^{4}+t^{2}\left(10+6 q^{2}\right)-t\left(20 q+4 q^{3}\right) \\
\hat{\mathcal{F}}_{s c 2}^{2}(q, t)= & 15+36 q^{2}-4 q^{3} t^{-3}-4 t^{3} q^{3}+16 q^{4}+q^{6}+\left(16 q^{2}+6 q^{4}\right) t^{-2}+ \\
& t^{2}\left(16 q^{2}+6 q^{4}\right)-\left(24 q+24 q^{3}+4 q^{5}\right) t^{-1}-t\left(24 q+24 q^{3}+4 q^{5}\right) \\
\hat{\mathcal{F}}_{s c 2}^{3}(q, t)= & -4 q t^{-3}+15 q^{2}-4 t q^{3}+q^{4}+\left(10+6 q^{2}\right) t^{-2}-\left(20 q+4 q^{3}\right) t^{-1} \\
\hat{\mathcal{F}}_{s c 2}^{4}(q, t)= & 4 t^{3} q^{3}-6 q^{4} t^{-2}-t^{2} q\left(16 q+6 q^{3}\right)+q\left(24 q^{2}+4 q^{4}\right) t^{-1}+ \\
& t q\left(20+24 q^{2}+4 q^{4}\right)-q\left(35 q+16 q^{3}+q^{5}\right) \\
\hat{\mathcal{F}}_{s c 2}^{5}(q, t)= & 4 q^{3} t^{-3}-6 t^{2} q^{4}-q\left(16 q+6 q^{3}\right) t^{-2}+q\left(20+24 q^{2}+4 q^{4}\right) t^{-1}+ \\
& t q\left(24 q^{2}+4 q^{4}\right)-q\left(35 q+16 q^{3}+q^{5}\right) \\
\hat{\mathcal{F}}_{s c 2}^{6}(q, t)= & 6 q^{4} t^{-2}+6 t^{2} q^{4}-q^{2}\left(20 q+4 q^{3}\right) t^{-1}-t q^{2}\left(20 q+4 q^{3}\right)+ \\
& q^{2}\left(20+15 q^{2}+q^{4}\right)
\end{aligned}
$$

For $(\ell-2,0,0,2)$ one has:

$$
\begin{aligned}
\hat{\mathcal{F}}_{s c 3}^{1}(q, t)= & t^{4}\left(q^{2}-q^{4}\right) \\
\hat{\mathcal{F}}_{s c 3}^{2}(q, t)= & q^{2}-q^{4} \\
\hat{\mathcal{F}}_{s c 3}^{3}(q, t)= & \left(q^{2}-q^{4}\right) t^{-4} \\
\hat{\mathcal{F}}_{s c 3}^{4}(q, t)= & 6 t^{2} q^{2}-4 t q^{3}-4 t^{3} q^{3}+q^{4}+t^{4} q^{4} \\
\hat{\mathcal{F}}_{s c 3}^{5}(q, t)= & 6 q^{2} t^{-2}-4 q^{3} t^{-3}-4 q^{3} t^{-1}+q^{4}+q^{4} t^{-4} \\
\hat{\mathcal{F}}_{s c 3}^{6}(q, t)= & 6 q^{4} t^{-2}+6 t^{2} q^{4}-q^{2}\left(20 q+4 q^{3}\right) t^{-1}-t q^{2}\left(20 q+4 q^{3}\right)+ \\
& q^{2}\left(20+15 q^{2}+q^{4}\right)
\end{aligned}
$$

For $(\ell, 0,0,1)$ one has:

$$
\begin{aligned}
& \hat{\mathcal{F}}_{g r 1}^{1}(q, t)=t^{2}\left(1-q^{2}\right) \\
& \hat{\mathcal{F}}_{g r 1}^{2}(q, t)=t^{-2}\left(1-q^{2}\right) \\
& \hat{\mathcal{F}}_{g r 1}^{3}(q, t)=6-4 q t^{-1}-4 t q+q^{2} t^{-2}+t^{2} q^{2}
\end{aligned}
$$

For $(\ell-1,0,1,0)$ one has:

$$
\begin{aligned}
& \hat{\mathcal{F}}_{g r 2}^{1}(q, t)=4 t q-6 q^{2}-t^{2} q^{2}+4 q^{3} t^{-1}-q^{4} t^{-2} \\
& \hat{\mathcal{F}}_{g r 2}^{2}(q, t)=4 q t^{-1}-6 q^{2}-q^{2} t^{-2}+4 t q^{3}-t^{2} q^{4} \\
& \hat{\mathcal{F}}_{g r 2}^{3}(q, t)=6 q^{2}-4 q^{3} t^{-1}-4 t q^{3}+q^{4} t^{-2}+t^{2} q^{4}
\end{aligned}
$$

For $(\ell-1,1,1,0)$ one has:

$$
\begin{aligned}
\hat{\mathcal{F}}_{f 1}^{1}(q, t)= & 4 t^{5} q^{3}-6 q^{4}-t^{4} q\left(16 q+6 q^{3}\right)+t q\left(24 q^{2}+4 q^{4}\right)+ \\
& t^{3} q\left(20+24 q^{2}+4 q^{4}\right)-t^{2} q\left(35 q+16 q^{3}+q^{5}\right)
\end{aligned}
$$




$$
\begin{aligned}
\hat{\mathcal{F}}_{f 1}^{2}(q, t)= & 4 q^{3} t^{-1}-6 t^{4} q^{4}-q\left(16 q+6 q^{3}\right)+t q\left(20+24 q^{2}+4 q^{4}\right)+ \\
& t^{3} q\left(24 q^{2}+4 q^{4}\right)-t^{2} q\left(35 q+16 q^{3}+q^{5}\right) \\
\hat{\mathcal{F}}_{f 1}^{3}(q, t)= & 4 q^{3} t^{-5}-6 q^{4}-q\left(16 q+6 q^{3}\right) t^{-4}+q\left(20+24 q^{2}+4 q^{4}\right) t^{-3}+ \\
& q\left(24 q^{2}+4 q^{4}\right) t^{-1}-q\left(35 q+16 q^{3}+q^{5}\right) t^{-2} \\
\hat{\mathcal{F}}_{f 1}^{4}(q, t)= & -15 q^{4} t^{-2}-10 t^{4} q^{4}+q\left(56 q^{2}+24 q^{4}\right) t^{-1}+t^{3} q\left(40 q^{2}+20 q^{4}\right)- \\
& t^{2} q\left(60 q+80 q^{3}+15 q^{5}\right)-q\left(74 q+90 q^{3}+16 q^{5}\right)+t q\left(36+120 q^{2}+60 q^{4}+4 q^{6}\right) \\
\hat{\mathcal{F}}_{f 1}^{5}(q, t)= & 4 t q^{3}-6 q^{4} t^{-4}-q\left(16 q+6 q^{3}\right)+q\left(24 q^{2}+4 q^{4}\right) t^{-3}+ \\
& q\left(20+24 q^{2}+4 q^{4}\right) t^{-1}-q\left(35 q+16 q^{3}+q^{5}\right) t^{-2} \\
\hat{\mathcal{F}}_{f 1}^{6}(q, t)= & -10 q^{4} t^{-4}-15 t^{2} q^{4}+t q\left(56 q^{2}+24 q^{4}\right)+q\left(40 q^{2}+20 q^{4}\right) t^{-3}- \\
& q\left(60 q+80 q^{3}+15 q^{5}\right) t^{-2}-q\left(74 q+90 q^{3}+16 q^{5}\right)+ \\
& q\left(36+120 q^{2}+60 q^{4}+4 q^{6}\right) t^{-1} \\
\hat{\mathcal{F}}_{f 1}^{7}(q, t)= & 4 t q-6 q^{2}-t^{2} q^{2}+4 q^{3} t^{-1}-q^{4} t^{-2} \\
\hat{\mathcal{F}}_{f 1}^{8}(q, t)= & 4 q t^{-1}-6 q^{2}-q^{2} t^{-2}+4 t q^{3}-t^{2} q^{4} \\
\hat{\mathcal{F}}_{f 1}^{9}(q, t)= & 6 q^{4}+6 t^{4} q^{4}-t q^{2}\left(20 q+4 q^{3}\right)-t^{3} q^{2}\left(20 q+4 q^{3}\right)+ \\
& t^{2} q^{2}\left(20+15 q^{2}+q^{4}\right) \\
\hat{\mathcal{F}}_{f 1}^{10}(q, t)= & 6 q^{4}+6 q^{4} t^{-4}-q^{2}\left(20 q+4 q^{3}\right) t^{-3}-q^{2}\left(20 q+4 q^{3}\right) t^{-1}+ \\
& q^{2}\left(20+15 q^{2}+q^{4}\right) t^{-2} \\
\hat{\mathcal{F}}_{f 1}^{11}(q, t)= & 6 q^{2}-4 q^{3} t^{-1}-4 t q^{3}+q^{4} t^{-2}+t^{2} q^{4}
\end{aligned}
$$

Finally, for $(\ell-2,1,0,1)$ one has:

$$
\begin{aligned}
\hat{\mathcal{F}}_{f 2}^{1}(q, t)= & 6 t^{4} q^{2}-4 t^{3} q^{3}-4 t^{5} q^{3}+t^{2} q^{4}+t^{6} q^{4} \\
\hat{\mathcal{F}}_{f 2}^{2}(q, t)= & 6 q^{2}-4 q^{3} t^{-1}-4 t q^{3}+q^{4} t^{-2}+t^{2} q^{4} \\
\hat{\mathcal{F}}_{f 2}^{3}(q, t)= & 6 q^{2} t^{-4}-4 q^{3} t^{-5}-4 q^{3} t^{-3}+q^{4} t^{-6}+q^{4} t^{-2} \\
\hat{\mathcal{F}}_{f 2}^{4}(q, t)= & t^{2}\left(q^{2}-q^{4}\right) \\
\hat{\mathcal{F}}_{f 2}^{5}(q, t)= & \left(q^{2}-q^{4}\right) t^{-2} \\
\hat{\mathcal{F}}_{f 2}^{6}(q, t)= & 4 t q+4 t^{3} q-6 q^{2}-6 t^{4} q^{2}+4 q^{3} t^{-1}+4 t^{5} q^{3}- \\
& q^{4} t^{-2}-t^{6} q^{4}-t^{2}\left(1+q^{2}\right) \\
\hat{\mathcal{F}}_{f 2}^{7}(q, t)= & 4 q t^{-3}+4 q t^{-1}-6 q^{2}-6 q^{2} t^{-4}+4 q^{3} t^{-5}+4 t q^{3}- \\
& q^{4} t^{-6}-t^{2} q^{4}-\left(1+q^{2}\right) t^{-2} \\
\hat{\mathcal{F}}_{f 2}^{8}(q, t)= & 6 q^{4}+6 t^{4} q^{4}-t q^{2}\left(20 q+4 q^{3}\right)-t^{3} q^{2}\left(20 q+4 q^{3}\right)+ \\
& t^{2} q^{2}\left(20+15 q^{2}+q^{4}\right) \\
\hat{\mathcal{F}}_{f 2}^{9}(q, t)= & 6 q^{4}+6 q^{4} t^{-4}-q^{2}\left(20 q+4 q^{3}\right) t^{-3}-q^{2}\left(20 q+4 q^{3}\right) t^{-1}+ \\
& q^{2}\left(20+15 q^{2}+q^{4}\right) t^{-2} \\
\hat{\mathcal{F}}_{f 2}^{10}(q, t)= & -6-32 q^{2}-12 q^{4}-6 q^{4} t^{-4}-6 t^{4} q^{4}+\left(24 q^{3}+4 q^{5}\right) t^{-3}+
\end{aligned}
$$




$$
\begin{aligned}
& t^{3}\left(24 q^{3}+4 q^{5}\right)+\left(24 q+28 q^{3}+4 q^{5}\right) t^{-1}+t\left(24 q+28 q^{3}+4 q^{5}\right)- \\
&\left(36 q^{2}+16 q^{4}+q^{6}\right) t^{-2}-t^{2}\left(36 q^{2}+16 q^{4}+q^{6}\right) \\
& \hat{\mathcal{F}}_{f 2}^{11}(q, t)=6 q^{2}-4 q^{3} t^{-1}-4 t q^{3}+q^{4} t^{-2}+t^{2} q^{4}
\end{aligned}
$$

Open Access. This article is distributed under the terms of the Creative Commons Attribution Noncommercial License which permits any noncommercial use, distribution, and reproduction in any medium, provided the original author(s) and source are credited.

\section{References}

[1] J.H. Schwarz, Superconformal Chern-Simons theories, JHEP 11 (2004) 078 [hep-th/0411077] [SPIRES].

[2] J. Bagger and N. Lambert, Modeling multiple M2's, Phys. Rev. D 75 (2007) 045020 [hep-th/0611108] [SPIRES].

[3] J. Bagger and N. Lambert, Gauge symmetry and supersymmetry of multiple M2-branes, Phys. Rev. D 77 (2008) 065008 [arXiv:0711.0955] [SPIRES].

[4] J. Bagger and N. Lambert, Comments on multiple M2-branes, JHEP 02 (2008) 105 [arXiv:0712.3738] [SPIRES].

[5] J. Bagger and N. Lambert, Three-algebras and $N=6$ Chern-Simons gauge theories, Phys. Rev. D 79 (2009) 025002 [arXiv:0807.0163] [SPIRES].

[6] A. Gustavsson, Algebraic structures on parallel M2-branes, Nucl. Phys. B 811 (2009) 66 [arXiv: 0709.1260] [SPIRES].

[7] O. Aharony, O. Bergman, D.L. Jafferis and J. Maldacena, $N=6$ superconformal Chern-Simons-matter theories, M2-branes and their gravity duals, JHEP 10 (2008) 091 [arXiv: 0806.1218] [SPIRES].

[8] I.R. Klebanov and G. Torri, M2-branes and AdS/CFT, Int. J. Mod. Phys. A 25 (2010) 332 [arXiv:0909.1580] [SPIRES].

[9] D.L. Jafferis and A. Tomasiello, A simple class of $N=3$ gauge/gravity duals, JHEP 10 (2008) 101 [arXiv:0808.0864] [SPIRES].

[10] D.L. Jafferis and X. Yin, Chern-Simons-Matter theory and mirror symmetry, arXiv:0810.1243 [SPIRES].

[11] D. Gaiotto and D.L. Jafferis, Notes on adding D6 branes wrapping RP3 in $A d S_{4} \times C P^{3}$, arXiv:0903.2175 [SPIRES].

[12] M. Aganagic, A stringy origin of M2 brane Chern-Simons theories, Nucl. Phys. B 835 (2010) 1 [arXiv:0905.3415] [SPIRES].

[13] D. Forcella and A. Zaffaroni, N=1 Chern-Simons theories, orientifolds and Spin(7) cones, JHEP 05 (2010) 045 [arXiv:0911.2595] [SPIRES]. 
[14] D.L. Jafferis, Quantum corrections to $N=2$ Chern-Simons theories with flavor and their $A d S_{4}$ duals, arXiv:0911.4324 [SPIRES].

[15] J. Bhattacharya, S. Bhattacharyya, S. Minwalla and S. Raju, Indices for superconformal field theories in 3,5 and 6 dimensions, JHEP 02 (2008) 064 [arXiv:0801.1435] [SPIRES].

[16] J. Bhattacharya and S. Minwalla, Superconformal Indices for $\mathcal{N}=6$ Chern Simons theories, JHEP 01 (2009) 014 [arXiv:0806.3251] [SPIRES].

[17] S. Kim, The complete superconformal index for $N=6$ Chern-Simons theory, Nucl. Phys. B 821 (2009) 241 [arXiv:0903.4172] [SPIRES].

[18] S. Kim and J. Park, Probing AdS4/CFT3 proposals beyond chiral rings, JHEP 08 (2010) 069 [arXiv: 1003.4343] [SPIRES].

[19] L. Castellani, R. D’Auria, P. Fré, K. Pilch and P. van Nieuwenhuizen, The bosonic mass formula for Freund-Rubin solutions of $D=11$ supergravity on general coset manifolds, Class. Quant. Grav. 1 (1984) 339 [SPIRES].

[20] A. Salam and E. Sezgin, Supergravities in diverse dimensions. Vol. 1-2, North-Holland, Amsterdam, Netherlands (1989) pg. 1499, World Scientific, Singapore, Singapore (1989) pg. 1499 [SPIRES].

[21] J.M. Maldacena, The large-N limit of superconformal field theories and supergravity, Adv. Theor. Math. Phys. 2 (1998) 231 [Int. J. Theor. Phys. 38 (1999) 1113] [hep-th/9711200] [SPIRES].

[22] O. Aharony, O. Bergman and D.L. Jafferis, Fractional M2-branes, JHEP 11 (2008) 043 [arXiv:0807.4924] [SPIRES].

[23] D. Gaiotto and A. Tomasiello, The gauge dual of Romans mass, JHEP 01 (2010) 015 [arXiv:0901.0969] [SPIRES].

[24] D. Gaiotto and A. Tomasiello, Perturbing gauge/gravity duals by a Romans mass, J. Phys. A 42 (2009) 465205 [arXiv:0904.3959] [SPIRES].

[25] M. Petrini and A. Zaffaroni, N=2 solutions of massive type IIA and their Chern-Simons duals, JHEP 09 (2009) 107 [arXiv:0904.4915] [SPIRES].

[26] B.E.W. Nilsson and C.N. Pope, Hopf fibration of eleven-dimensional supergravity, Class. Quant. Grav. 1 (1984) 499 [SPIRES].

[27] I. Klebanov, T. Klose and A. Murugan, $A d S_{4} / C F T_{3}$ - Squashed, stretched and warped, JHEP 03 (2009) 140 [arXiv:0809.3773] [SPIRES].

[28] M.K. Benna, I.R. Klebanov and T. Klose, Charges of monopole operators in Chern-Simons Yang-Mills theory, JHEP 01 (2010) 110 [arXiv:0906.3008] [SPIRES].

[29] P. Van Nieuwenhuizen, An introduction to simple supergravity and the Kaluza-Klein program, in proceedings of Relativity, Groups and Topology, Les Houches (1983) pg. 823 [SPIRES].

[30] E. Sezgin, The spectrum of the eleven-dimensional supergravity compactified on the round seven sphere, Phys. Lett. B 138 (1984) 57 [SPIRES].

[31] D. P. Zhelobenko Compact lie groups and their representations, Translations of mathematical monographs, Vol. 40 (1973). 
[32] M. Benna, I. Klebanov, T. Klose and M. Smedback, Superconformal Chern-Simons theories and $A d S_{4} / C F T_{3}$ correspondence, JHEP 09 (2008) 072 [arXiv:0806.1519] [SPIRES].

[33] A. Cagnazzo, D. Sorokin and L. Wulff, String instanton in $A d S_{4} \times C P^{3}$, JHEP 05 (2010) 009 [arXiv:0911.5228] [SPIRES].

[34] K. Hosomichi et al., A nonperturbative test of M2-brane theory, JHEP 11 (2008) 058 [arXiv: 0809.1771] [SPIRES].

[35] M. Bianchi, Bound-states of D-branes in L-R asymmetric superstring vacua, Nucl. Phys. B 805 (2008) 168 [arXiv:0805.3276] [SPIRES].

[36] M. Bianchi, From twists and shifts to L-R asymmetric D-branes, Fortsch. Phys. 57 (2009) 356 [arXiv:0902.0650] [SPIRES].

[37] M. Bianchi and M. Samsonyan, Notes on unoriented D-brane instantons, Int. J. Mod. Phys. A 24 (2009) 5737 [arXiv:0909.2173] [SPIRES].

[38] E. Sezgin and P. Sundell, Higher spin $N=8$ supergravity, JHEP 11 (1998) 016 [hep-th/9805125] [SPIRES].

[39] E. Sezgin and P. Sundell, Massless higher spins and holography, Nucl. Phys. B 644 (2002) 303 [Erratum ibid. B 660 (2003) 403] [hep-th/0205131] [SPIRES].

[40] J. Engquist, E. Sezgin and P. Sundell, On $N=1,2,4$ higher spin gauge theories in four dimensions, Class. Quant. Grav. 19 (2002) 6175 [hep-th/0207101] [SPIRES].

[41] M.A. Vasiliev, Higher spin gauge theories: Star-product and AdS space, hep-th/9910096 [SPIRES].

[42] M. Bianchi, Higher spin symmetry (breaking) in $N=4$ SYM and holography, Comptes Rendus Physique 5 (2004) 1091 [hep-th/0409292] [SPIRES].

[43] M. Bianchi, Higher spins and stringy $A d S_{5} \times S^{5}$, Fortsch. Phys. 53 (2005) 665 [hep-th/0409304] [SPIRES].

[44] X. Bekaert, S. Cnockaert, C. Iazeolla and M.A. Vasiliev, Nonlinear higher spin theories in various dimensions, hep-th/0503128 [SPIRES].

[45] D. Francia and A. Sagnotti, Higher-spin geometry and string theory, J. Phys. Conf. Ser. 33 (2006) 57 [hep-th/0601199] [SPIRES].

[46] S. Ferrara and C. Fronsdal, Conformal Maxwell theory as a singleton field theory on AdS $S_{5}$, IIB three branes and duality, Class. Quant. Grav. 15 (1998) 2153 [hep-th/9712239] [SPIRES].

[47] B. Sundborg, The hagedorn transition, deconfinement and $N=4$ SYM theory, Nucl. Phys. B 573 (2000) 349 [hep-th/9908001] [SPIRES].

[48] P. Haggi-Mani and B. Sundborg, Free large- $N$ supersymmetric Yang-Mills theory as a string theory, JHEP 04 (2000) 031 [hep-th/0002189] [SPIRES].

[49] M. Bianchi, S. Kovacs, G. Rossi and Y.S. Stanev, On the logarithmic behavior in $N=4$ SYM theory, JHEP 08 (1999) 020 [hep-th/9906188] [SPIRES]. 
[50] M. Bianchi, S. Kovacs, G. Rossi and Y.S. Stanev, Anomalous dimensions in $N=4 S Y M$ theory at order $g^{4}$, Nucl. Phys. B 584 (2000) 216 [hep-th/0003203] [SPIRES].

[51] M. Bianchi, S. Kovacs, G. Rossi and Y.S. Stanev, Properties of the Konishi multiplet in $N=4$ SYM theory, JHEP 05 (2001) 042 [hep-th/0104016] [SPIRES].

[52] M. Bianchi, J.F. Morales and H. Samtleben, On stringy $A d S_{5} \times S^{5}$ and higher spin holography, JHEP 07 (2003) 062 [hep-th/0305052] [SPIRES].

[53] N. Beisert, M. Bianchi, J.F. Morales and H. Samtleben, On the spectrum of AdS/CFT beyond supergravity, JHEP 02 (2004) 001 [hep-th/0310292] [SPIRES].

[54] N. Beisert, M. Bianchi, J.F. Morales and H. Samtleben, Higher spin symmetry and $N=4$ SYM, JHEP 07 (2004) 058 [hep-th/0405057] [SPIRES].

[55] M. Bianchi, P.J. Heslop and F. Riccioni, More on la grande bouffe, JHEP 08 (2005) 088 [hep-th/0504156] [SPIRES].

[56] F.A. Dolan, On superconformal characters and partition functions in three dimensions, J. Math. Phys. 51 (2010) 022301 [arXiv:0811.2740] [SPIRES].

[57] M. Bianchi, F.A. Dolan, P.J. Heslop and H. Osborn, $N=4$ superconformal characters and partition functions, Nucl. Phys. B 767 (2007) 163 [hep-th/0609179] [SPIRES].

[58] C. Kounnas, D. Lüst, P.M. Petropoulos and D. Tsimpis, AdS $S_{4}$ flux vacua in type-II superstrings and their domain- wall solutions, JHEP 09 (2007) 051 [arXiv:0707.4270] [SPIRES].

[59] C. Caviezel et al., The effective theory of type IIA AdS $S_{4}$ compactifications on nilmanifolds and cosets, Class. Quant. Grav. 26 (2009) 025014 [arXiv:0806.3458] [SPIRES].

[60] M. Haack, D. Lüst, L. Martucci and A. Tomasiello, Domain walls from ten dimensions, JHEP 10 (2009) 089 [arXiv:0905.1582] [SPIRES].

[61] M. Bianchi and W. de Paula, On exact symmetries and massless vectors in holographic flows and other flux vacua, JHEP 04 (2010) 113 [arXiv: 1003.2536] [SPIRES]. 